\title{
MIMO Full-Duplex Relaying in the Presence of Co-Channel Interference
}

DOI:

10.1109/TVT.2016.2616946

\section{Document Version}

Accepted author manuscript

Link to publication record in Manchester Research Explorer

\section{Citation for published version (APA):}

Almradi, A., \& Hamdi, K. (2016). MIMO Full-Duplex Relaying in the Presence of Co-Channel Interference. IEEE Transactions on Vehicular Technology. https://doi.org/10.1109/TVT.2016.2616946

\section{Published in:}

IEEE Transactions on Vehicular Technology

\section{Citing this paper}

Please note that where the full-text provided on Manchester Research Explorer is the Author Accepted Manuscript or Proof version this may differ from the final Published version. If citing, it is advised that you check and use the publisher's definitive version.

\section{General rights}

Copyright and moral rights for the publications made accessible in the Research Explorer are retained by the authors and/or other copyright owners and it is a condition of accessing publications that users recognise and abide by the legal requirements associated with these rights.

\section{Takedown policy}

If you believe that this document breaches copyright please refer to the University of Manchester's Takedown Procedures [http://man.ac.uk/04Y6Bo] or contact uml.scholarlycommunications@manchester.ac.uk providing relevant details, so we can investigate your claim.

\section{OPEN ACCESS}




\title{
MIMO Full-Duplex Relaying in the Presence of Co-Channel Interference
}

\author{
Ahmed Almradi, Student Member, IEEE, and Khairi Ashour Hamdi, Senior Member, IEEE
}

\begin{abstract}
This paper studies the deployment of multiple-input multiple-output (MIMO) full-duplex (FD) relaying systems in a multi-cell environment, where the source and destination nodes are equipped with single antenna and communicating via a dualhop amplify-and-forward (AF) relay station with multiple receive and transmit antennas in the presence of co-channel interference (CCI). This paper addresses the fundamental challenges of loopback self-interference (LI) and CCI when incorporating FD relaying in cellular systems. Due to the higher frequency reuse in FD relaying compared to its half-duplex (HD) relaying counterpart, the CCI is expected to double as the FD relay station simultaneously schedule uplink and downlink transmission on the same channel. The optimal design of relay receive and transmit precoding weight vectors which maximizes the overall signal-to-interefernce-plus-noise ratio (SINR) is formulated by a proper optimization problem, then a closed-form suboptimal solution based on null space projection is proposed. The proposed precoding vectors are based on the added receive and transmit zero-forcing ( $\mathrm{ZF})$ constraints used to suppress the co-channel interference and loopback self-interfernce, respectively. To this end, exact closed-form expressions for the outage probability and ergodic capacity are derived, where a simpler lower-bound expressions are also presented. In addition, the asymptotic high signal-to-noise ratio (SNR) outage probability approximation is also considered, through which the diversity order of the null space projection (ZF/ZF) scheme is found to achieve $\min \left(N_{R}-M, N_{T}-1\right)$, where $N_{R}$ and $N_{T}$ are the number of relay receive and transmit antennas, respectively, and $M$ is the number of CCI interferers. Numerical results sustained by Monte-Carlo simulations show the exactness of the proposed analytical expressions as well as the tightness of the proposed lower-bound expressions. In addition, simulation results for the minimum mean square error (MMSE)/ZF scheme is also considered for comparison purposes. Our results reveal that MIMO FD relaying could substantially boost the system performance compared to its conventional MIMO HD relaying counterpart.
\end{abstract}

Index Terms-MIMO relaying, full-duplex relaying, halfduplex relaying, zero-forcing $(\mathrm{ZF})$, minimum mean square error (MMSE), outage probability, ergodic capacity, co-channel interference.

\section{INTRODUCTION}

$\mathbf{C}$ OOPERATIVE relaying techniques have recently gained a great deal of attention due to their ability to extend network coverage, connectivity and attain higher capacity without

Copyright (c) 2015 IEEE. Personal use of this material is permitted. However, permission to use this material for any other purposes must be obtained from the IEEE by sending a request to pubs-permissions@ieee.org.

A. Almradi is with the Department of Electrical and Electronic Engineering, Azzaytuna University, Tarhuna, Libya. He is also with the school of Electrical and Electronic Engineering, The University of Manchester, Manchester, M13 9PL, UK. (e-mail: elmaradi@gmail.com).

K. A. Hamdi is with the school of Electrical and Electronic Engineering, The University of Manchester, Manchester, M13 9PL, UK. (e-mail: k.hamdi@manchester.ac.uk). sacrificing extra power resources. In a dual-hop relaying systems, an intermediate idle node operates as a relay between the source and destination nodes when the direct link between the source and destination is in deep fade. Two orthogonal channels are required for communications to take place in the conventional dual-hop one-way relaying networks. As a result, a significant loss of spectrum efficiency is incurred because of the inherent half-duplex (HD) relaying transmission constraint at the relay, where time-division duplex or frequency-division duplex is used by the conventional wireless communication systems to operate in out-of-band full-duplex (half-duplex). Recently, full-duplex (FD) relaying, also known as in-band full-duplex, has received a lot of research interest because of its potential to double spectral efficiency (e.g., [1]-[14]). This is due to the fact that full-duplex relays receive and re-transmit its information at the same time over the same frequency. Hence, efficiently utilizing the spectrum resources of the system. However, the main limitation of FD relaying is the loopback self-interference (LI) caused by the signal leakage from the relay's transmission to its own reception, namely, the large power differences between the LI (power transmitted from the FD relay) and the FD relay received signal (which is much weaker than the transmitted signal due to path loss and fading) exceeds the dynamic range of the analoge-to-digital converter. Therefore, LI mitigation and cancellation is vital in the implementation of FD relaying operation [1]-[6], [15].

Linking multiple-input-multiple-output (MIMO) techniques with full-duplex relaying systems provide a powerful capability of suppressing LI in the spatial domain and can offer higher capacity when compared to its MIMO HD relaying counterpart (see e.g., [10], [11], [16], [17]). Therefore, in the presence of MIMO FD relaying systems, joint optimization by decoding and precoding at the receiver and transmitter, respectively, can be used to mitigate the LI effects at the relay. Due to its implementation simplicity and mathematical tractability, zeroforcing (ZF) technique is utilized to completely cancel out the LI interference and disconnect the closed-loop between the relay's transmission and reception. Numerous papers have studied the spatial LI mitigation problem, for instance, ZF precoding and decoding vectors based on the conventional singular value decomposition (SVD) of the LI channel is proposed in [10], [16] to null out the LI at the relay, where [10] presented different spatial LI suppression techniques, namely, antenna selection, beam selection, and null space projection. In addition, a low complexity joint precoding/decoding design for maximizing the overall signal-to-noise ratio (SNR) is investigated in [11], where a closed-form overall SNR is derived. More specifically, both receive ZF precoding with 
maximum-ratio transmission (MRT) scheme, and maximalratio combining (MRC) with transmit $\mathrm{ZF}$ scheme have been presented. More recently, a massive receive antenna array or a massive transmit antenna array is used to significantly reduce the LI effect is introduced in [9], where ZF/ZF scheme or MRC/MRT scheme is used at the FD relay. In [18], different optimaization problems which maximaizes the overall SNR of MIMO FD relaying are inestigated. The authors in [19] analyzed the performance of MIMO FD relaying when null space projection via SVD is used to mitigate the LI interference. In [20], the degrees of freedom of bidirectional interference network is considered by utilizing interference alignment, assuming that the LI is known to the receiver and therefore can be subtracted off completely. However, to the best of our knowledge, the impact of co-channel interference $(\mathrm{CCI})^{1}$ on the performance of MIMO full-duplex relaying has not been investigated yet.

Due to the aggressive frequency reuse in the conventional HD cellular relaying systems, the impact of CCI on the system performance has been extensively studied in the literature (see e.g., [21]-[23]). However, FD cellular relaying systems are more vulnerable to CCI owing to the higher frequency reuse compared to its traditional HD relaying counterpart. For instance, in a multi-cell environment with FD relay base stations and HD users, a much higher CCI is seen from adjacent cells as compared to its HD counterpart [2], [24]-[26]. Therefore, analyzing the detrimental effect of CCI on the performance of MIMO FD relaying transmission is of theoretical and practical importance. In [25], [26], the performance analysis of FD relaying subject to CCI have been investigated, namely, the outage probability of a decode-and-forward FD relay with single antenna nodes have been presented. In [24], the average spectral efficiency of a stochastic geometry small cell network, where the base stations and user equipments operate in fullduplex mode (i.e., nodes have dedicated antennas for transmission and reception) is investigated. It is to be emphasized that all these works are limited to single antenna nodes. However, the deployment of multiple receive and transmit antennas at the FD relay not only improve reliability and increase capacity, it yields a powerful technique for suppressing both, the CCI and LI channels at the FD relay.

Motivated by the above mentioned limitations, this paper investigates the impact of co-channel interference on the performance of MIMO amplify-and-forward FD relaying systems with ZF/ZF scheme, where its performance is compared against simulation results for the minimum mean square error (MMSE)/ZF scheme.

The main contributions of this paper are summarized as follows:

1) The optimization problem for the design of relay receive and transmit precoding weight vectors that maximizes the overall signal-to-interefernce-plus-noise ratio (SINR) is formulated, then a sub-optimal solution based on

\footnotetext{
${ }^{1}$ The frequency resue deployment to enhance the spectrum efficiency of wireless systems cause the harmful effect of CCI in the HD multi-cell environment case, where a greater frequency resue is seen in the FD multicell environment case which causes the challenge of doubling CCI in the neighboring cells.
}

the added receive and transmit zero-forcing (null space projection) constraints is proposed, through which a sub-optimal precoding and decoding weight vectors are presented. The receive and transmit ZF precoding weight vectors ( $\mathrm{ZF} / \mathrm{ZF}$ scheme) is designed as follows; the receive $\mathrm{ZF}$ weight vector is designed to suppress the $\mathrm{CCI}$, while the transmit ZF weight vector is designed to null out the LI.

2) A new exact closed-form expression for the outage probability of MIMO FD relaying systems with CCI is presented for the derived overall SINR, where ZF/ZF scheme is applied. In addition, a simpler tight closedform lower-bound outage probability expression is also included. Besides, the asymptotic analysis of the system under consideration is also studied, where the characterization of high SNR outage probability show that the achievable diversity order is $\min \left(N_{R}-M, N_{T}-1\right)$, where $N_{R}$ and $N_{T}$ are the number of relay receive and transmit antennas, respectively, and $M$ is the number of CCI interferers.

3) A new exact closed-form ergodic capacity expression is derived for the proposed overall SINR, where a simpler tight closed-form lower-bound ergodic capacity expression is also presented.

The structure of the rest of the paper is as follows. In section II, we introduce the system model. In section III, the instantaneous overall SNR is addressed. In section IV, the outage probability analysis is considered. In section V, the ergodic capacity analysis is investigated. The MMSE/ZF scheme is introduced in section VI. Numerical results are provided in section VII. Finally, section VIII concludes the paper.

\section{The System Model}

We consider a MIMO full-duplex AF relaying system, where a single antenna source $\boldsymbol{S}$ is communicating with a single antenna destination $\boldsymbol{D}$ through a multi-antenna FD relay $\boldsymbol{R}$ with $N_{R}$ receiving antennas and $N_{T}$ transmitting antennas, where the relay is subject to loopback self-interference in addition to $M$ co-channel interferers (CCI) as depicted in Fig. 1. Throughout this paper, the following assumptions are considered: 1) As our main focus on this paper is on network coverage extension (see e.g., [18], [21], [27], [28]), it is assumed that the source does not have a direct link to the destination due to heavy path loss and shadowing ${ }^{2}$. 2) A single MIMO full-duplex AF relay is considered, where the full-duplex relay receive and re-transmit its information at the same time over the same frequency. 3) It is also assumed that the relay suffers $M \mathrm{CCI}$ interferers in addition to additive white Gaussian noise (AWGN), while the destination is corrupted by AWGN. The practical justification of such an assumption is that two mobile users which are far from the cell edge exchange information with the aid of a relay

\footnotetext{
${ }^{2}$ In the case of single antenna nodes and in the absence of CCI, the effect of the direct link between the source and destination on the system performance has been investigated in [27].
} 
that is close to the cell edge ${ }^{3}$. 4) Channels are modeled as quasi-static block flat fading and remain constant over the block time $T$, and varies independently and identically from one block to the next. The source $\rightarrow$ relay $(S \rightarrow R)$ channel $\boldsymbol{h}_{1}$ with $N_{R} \times 1$ vector, the relay $\rightarrow$ destination $(\boldsymbol{R} \rightarrow \boldsymbol{D})$ channel $\boldsymbol{h}_{2}$ with $N_{T} \times 1$ vector, the relay $\rightarrow$ relay $(\boldsymbol{R} \rightarrow \boldsymbol{R})$ residual LI channel $\boldsymbol{H}_{R}$, which models the resultant error due to imperfect LI mitigation performed by antennas isolation, and analog and digital cancellation at the FD relay, the $i^{\text {th }}$ interferer channel at the relay $\boldsymbol{g}_{i}$ with $N_{R} \times 1$ vector are modeled by independent Rayleigh fading with entries follow independent and identically distributed random variables $(i . i . d$.) with $\mathcal{C N}(0,1)$. Full channel state information (CSI) of the $\boldsymbol{S} \rightarrow \boldsymbol{R}$ channel $\boldsymbol{h}_{1}$, the $\boldsymbol{R} \rightarrow \boldsymbol{D}$ channel $\boldsymbol{h}_{2}$, the $\boldsymbol{R} \rightarrow \boldsymbol{R}$ residual LI channel $\boldsymbol{H}_{R}$, and the $i^{\text {th }}$ interferer channel $\boldsymbol{g}_{i}$ with $i=1, \ldots, M$ are assumed to be available at the relay for the $\mathrm{ZF} / \mathrm{ZF}$ scheme to be implemented. 5) In order to completely eliminate the CCI channel at the relay, the number of relay receive antennas is assumed to be greater than the number of interferers (i.e., $N_{R}>M$ ). This is a necessary condition for the deployment of receive $\mathrm{ZF}$ scheme ${ }^{4}$. Similarly, in order to completely eliminate the LI channel at the relay, the number of relay transmit antennas is supposed to be larger than one (i.e., $N_{T}>1$ ). This is an essential condition for ensuring the feasibility of transmit $\mathrm{ZF}$ scheme.

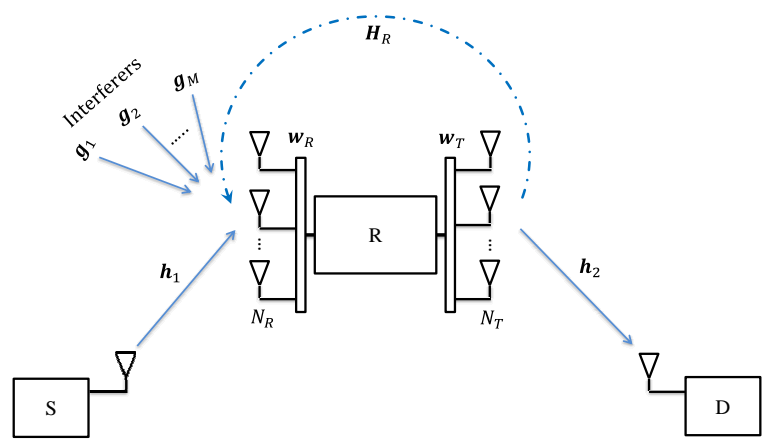

Figure 1: The MIMO FD relaying system model.

\section{The InStANTANEOUS OVERALL SNR}

The received signal at the relay after the receive precoding vector can be written as

$$
\boldsymbol{w}_{R}^{\dagger} \boldsymbol{y}_{R}=\boldsymbol{w}_{R}^{\dagger}\left(\boldsymbol{h}_{1} x_{S}+\boldsymbol{H}_{R} \boldsymbol{w}_{T} x_{R}+\sum_{i=1}^{M} \boldsymbol{g}_{i} x_{i}+\boldsymbol{n}_{R}\right)
$$

where $x_{S}$ is the source information signal with average power $\mathcal{E}_{S}=\mathbb{E}\left[x_{S} x_{S}^{\star}\right]$, with $\mathbb{E}(\cdot)$ denotes the expectation operator, $(\cdot)^{\star}$ denotes the conjugate operator, $x_{R}$ is the relay signal

\footnotetext{
${ }^{3}$ Note that our analysis can be easily extended to the case where the destination also suffers multiple CCI interferers. However, the purpose of this paper is to analyze the effect of MIMO FD relay on combating both, the $\mathrm{CCI}$ and LI interferences compared to its MIMO HD relaying couterpart. It is to be emphasized that users are operating in the HD mode while the relay is operating in the FD mode. Therefore, including CCI at the destination would only make the analysis complicated without any additional insights.

${ }^{4}$ In section (VI), the case of MMSE/ZF scheme is also considered for comparison purposes.
}

with average power $\mathcal{E}_{R}=\mathbb{E}\left[x_{R} x_{R}^{\star}\right], x_{i}$ is the $i^{\text {th }}$ interferer signal at the relay with average power $\mathcal{E}_{i}=\mathbb{E}\left[x_{i} x_{i}^{\star}\right]$, $\boldsymbol{n}_{R}$ is an $N_{R} \times 1$ vector which denotes the additive white Gaussian noise (AWGN) at the relay, distributed according to $\boldsymbol{n}_{R} \sim \mathcal{C N}\left(0, \sigma_{R}^{2} \boldsymbol{I}_{N_{R}}\right), \boldsymbol{w}_{R}$ is the receive beamforming weight vector, and $(\cdot)^{\dagger}$ denotes the conjugate transpose operator. Let $\boldsymbol{G}=\left[\boldsymbol{g}_{1}, \boldsymbol{g}_{2}, \ldots, \boldsymbol{g}_{M}\right]$ be the co-channel interference (CCI) matrix with dimension $N_{R} \times M$. Then, the term $\sum_{i=1}^{M} \boldsymbol{w}_{R}^{\dagger} \boldsymbol{g}_{i} x_{i}$ in (1) can be re-written as $\boldsymbol{w}_{R}^{\dagger} \boldsymbol{G} \boldsymbol{x}_{I}$ where $\boldsymbol{x}_{I}=\left[x_{1}, x_{2}, \ldots, x_{M}\right]^{T}$, with $(\cdot)^{T}$ denotes the transpose operator.

The received signal at the destination can be written as

$$
y_{D}=\sqrt{\mathcal{E}_{R}} \boldsymbol{h}_{2}^{\dagger} \boldsymbol{w}_{T} x_{R}+n_{D}
$$

where $\boldsymbol{w}_{T}$ is the transmit beamforming weight vector, and $n_{D}$ is the AWGN at the destination and distributed according to $n_{D} \sim \mathcal{C N}\left(0, \sigma_{D}^{2}\right)$.

From (1) and (2), the $\boldsymbol{S} \rightarrow \boldsymbol{R}$ SINR and $\boldsymbol{R} \rightarrow \boldsymbol{D}$ SNR (respectively) can be obtained as

$$
\gamma_{1}=\frac{\overline{\gamma_{1}}\left\|\boldsymbol{w}_{R}^{\dagger} \boldsymbol{h}_{1}\right\|^{2}}{\overline{\gamma_{2}}\left\|\boldsymbol{w}_{R}^{\dagger} \boldsymbol{H}_{R} \boldsymbol{w}_{T}\right\|^{2}+\frac{1}{\sigma_{R}^{2}} \mathbb{E}\left(\left\|\boldsymbol{w}_{R}^{\dagger} \boldsymbol{G} \boldsymbol{x}_{I}\right\|^{2}\right)+1}
$$

and

$$
\gamma_{2}=\overline{\gamma_{2}}\left\|\boldsymbol{h}_{2}^{\dagger} \boldsymbol{w}_{T}\right\|^{2}
$$

where $\overline{\gamma_{1}}=\frac{\mathcal{E}_{S}}{\sigma_{R}^{2}}, \overline{\gamma_{2}}=\frac{\mathcal{E}_{R}}{\sigma_{D}^{2}},\|\cdot\|$ denotes the Euclidean norm operator, and $\mathbb{E}\left(\left\|\boldsymbol{w}_{R}^{\dagger} \boldsymbol{G} \boldsymbol{x}_{I}\right\|^{2}\right)=\sum_{i=1}^{M}\left\|\boldsymbol{w}_{R}^{\dagger} \boldsymbol{g}_{i}\right\|^{2} \mathcal{E}_{i}$

Therefore, the overall SINR can be simplified to

$$
\gamma=\frac{\gamma_{1} \gamma_{2}}{\gamma_{1}+\gamma_{2}+1}
$$

where $\gamma_{1}$ is the first-hop SNR given in (3), and $\gamma_{2}$ is the second-hop SNR given in (4).

Our Objective is to find an optimal transmit and receive precoding vectors, $\left\{\boldsymbol{w}_{T}, \boldsymbol{w}_{R}\right\}$ so that the overall SINR in (5) is maximized. Therefore, the optimization problem may be formulated as

$$
\begin{array}{cc}
\boldsymbol{w}_{T}^{\star}, \boldsymbol{w}_{R}^{\star}=\underset{\boldsymbol{w}_{T}, \boldsymbol{w}_{R}}{\arg \max } & \gamma(\text { in Eq. (5)) } \\
\text { s.t. } & \left\|\boldsymbol{w}_{T}\right\|=\left\|\boldsymbol{w}_{R}\right\|=1
\end{array}
$$

It is well known that in the absence of interference, maximum-ratio combining (MRC) at the receiver and maximum-ratio transmission (MRT) at the transmitter are optimal precoding schemes as they result in the maximum overall SINR. However, due to the aggressive frequency reuse in FD relaying systems, through which a higher and deleterious effect of CCI is seen as it is expected to double compared to its conventional HD relaying counterpart [2], MRC and MRT are sub-optimal as they treat the interference as additive noise. Therefore, the presence of CCI can severely deteriorate the performance of FD relaying systems and its mitigation is of theoretical and practical interest. Owing to the complexity of the optimization problem in (6), the optimal receive and transmit precoding vectors at the MIMO FD relay are nontrivial 
to solve in closed-form. Therefore, as far as a closed-form simple analytically tractable overall SINR is concern, a suboptimal solution is found by adding a receive and transmit ZF constraints to the optimization problem in $(6)^{5}$. These constraints force the loopback self and co-channel interference terms at the MIMO FD relay to zero, i.e., $\boldsymbol{w}_{R}^{\dagger} \boldsymbol{H}_{R} \boldsymbol{w}_{T}=0$ and $\boldsymbol{w}_{R}^{\dagger} \boldsymbol{G}=0$, assuming that $N_{R}>M$ and $N_{T}>1$. To this end, due to the separability of the resultant constraint problem, the optimization problem could be separated into two simpler problems as follows

$$
\begin{array}{cc}
\boldsymbol{w}_{R}^{\star}=\underset{\boldsymbol{w}_{R}}{\arg \max } & \left\|\boldsymbol{w}_{R}^{\dagger} \boldsymbol{h}_{1}\right\|^{2} \\
\text { s.t. } & \boldsymbol{w}_{R}^{\dagger} \boldsymbol{G}=0 \&\left\|\boldsymbol{w}_{R}\right\|=1
\end{array} .
$$

and

$$
\begin{array}{cc}
\boldsymbol{w}_{T}^{\star}=\underset{\boldsymbol{w}_{T}}{\arg \max } & \left\|\boldsymbol{h}_{2}^{\dagger} \boldsymbol{w}_{T}\right\|^{2} \\
\text { s.t. } & \boldsymbol{w}_{R}^{\dagger} \boldsymbol{H}_{R} \boldsymbol{w}_{T}=0 \&\left\|\boldsymbol{w}_{T}\right\|=1
\end{array} .
$$

Proposition 1. The optimal solution to the constraint optimization problems in (7) and (8) are (respectively) derived as

$$
\boldsymbol{w}_{R}^{\star}=\frac{\boldsymbol{P} \boldsymbol{h}_{1}}{\left\|\boldsymbol{P} \boldsymbol{h}_{1}\right\|}
$$

where $\boldsymbol{P}=\boldsymbol{I}_{N_{R}}-\boldsymbol{G}\left(\boldsymbol{G}^{\dagger} \boldsymbol{G}\right)^{-1} \boldsymbol{G}^{\dagger}$, and

$$
\boldsymbol{w}_{T}^{\star}=\frac{\boldsymbol{B} \boldsymbol{h}_{2}}{\left\|\boldsymbol{B} \boldsymbol{h}_{2}\right\|}
$$

where $\boldsymbol{B}=\boldsymbol{I}_{N_{T}}-\frac{\boldsymbol{H}_{R}^{\dagger} \boldsymbol{P \boldsymbol { h } _ { 1 }}\left(\boldsymbol{H}_{R}^{\dagger} \boldsymbol{P} \boldsymbol{h}_{1}\right)^{\dagger}}{\left(\boldsymbol{H}_{R}^{\dagger} \boldsymbol{P} \boldsymbol{h}_{1}\right)^{\dagger} \boldsymbol{H}_{R}^{\dagger} \boldsymbol{P h} \boldsymbol{h}_{1}}$.

Proof: The proof is given in Appendix A.

Therefore, the overall SINR of MIMO FD relaying with CCI utilizing null-space projection (ZF/ZF scheme) can be derived as

$$
\gamma^{\mathrm{ZF} / \mathrm{ZF}}=\frac{\gamma_{1}^{\mathrm{ZF}} \gamma_{2}^{\mathrm{ZF}}}{\gamma_{1}^{\mathrm{ZF}}+\gamma_{2}^{\mathrm{ZF}}+1}
$$

where $\gamma_{1}^{\mathrm{ZF}}=\overline{\gamma_{1}}\left|\boldsymbol{h}_{1}^{\dagger} \boldsymbol{P} \boldsymbol{h}_{1}\right|=\overline{\gamma_{1}}\left\|\boldsymbol{P} \boldsymbol{h}_{1}\right\|^{2}, \gamma_{2}^{\mathrm{ZF}}=$ $\overline{\gamma_{2}}\left|\boldsymbol{h}_{2}^{\dagger} \boldsymbol{B} \boldsymbol{h}_{2}\right|=\overline{\gamma_{2}}\left\|\boldsymbol{B} \boldsymbol{h}_{2}\right\|^{2}$.

Note that the matrices $\boldsymbol{P}$ and $\boldsymbol{B}$ are idempotent orthogonal projection (null-space projection) matrices (all their eigenvalues are either one or zero, where the number of ones is determined by the rank of the matrix ${ }^{6}$ ) which are used to eliminate the co-channel interference and loopback selfinterference channel, respectively. For instance, in the case of idempotent orthogonal projection matrix $\boldsymbol{P}$, we have $\operatorname{tr}(\boldsymbol{P})=$ $\operatorname{rank}(\boldsymbol{P})$ because of idempotency, where $\operatorname{tr}(\cdot)$ denotes the

\footnotetext{
${ }^{5}$ It is to be emphasized that the asymptotic results of the ZF scheme is optimal, i.e., the ZF scheme is optimal in the high interferer-to-noise ratio (INR) regime. This is proved through simulation results of the MMSE/ZF scheme presented in section VI.

${ }^{6}$ It is well known that the rank of an $M \times N$ matrix $A$ is $\operatorname{rank}(A) \leq$ $\min (M, N)$, where equality holds if and only if $A$ is full rank.
}

trace operation. Hence, we have

$$
\begin{aligned}
\operatorname{rank}(\boldsymbol{P}) & =\operatorname{rank}\left(\boldsymbol{I}_{N_{R}}-\boldsymbol{G}\left(\boldsymbol{G}^{\dagger} \boldsymbol{G}\right)^{-1} \boldsymbol{G}^{\dagger}\right) \\
& =\operatorname{rank}\left(\boldsymbol{I}_{N_{R}}\right)-\operatorname{rank}\left(\boldsymbol{G}\left(\boldsymbol{G}^{\dagger} \boldsymbol{G}\right)^{-1} \boldsymbol{G}^{\dagger}\right) .
\end{aligned}
$$

It can be easily shown that $\operatorname{rank}\left(\boldsymbol{I}_{N_{R}}\right)=N_{R}$ and $\operatorname{rank}\left(\boldsymbol{G}\left(\boldsymbol{G}^{\dagger} \boldsymbol{G}\right)^{-1} \boldsymbol{G}^{\dagger}\right)=\operatorname{rank}(\boldsymbol{G})=M$. Hence, $\operatorname{rank}(\boldsymbol{P})=N_{R}-M$.

Therefore, the vector $\boldsymbol{P} \boldsymbol{h}_{1}$ has the same statistics as $\boldsymbol{h}_{1}$ with dimensionality reduced by the number of interferers $M$, hence $\boldsymbol{P} \boldsymbol{h}_{1} \sim \mathcal{C N}\left(0, \boldsymbol{I}_{N_{R}-M}\right)$. Similarly, it can be shown that the vector $\boldsymbol{B} \boldsymbol{h}_{2}$ has the same statistics as $\boldsymbol{h}_{2}$ with dimensionality reduced by one (i.e., $\operatorname{rank}(\boldsymbol{B})=N_{T}-$ $\operatorname{rank}\left(\boldsymbol{H}_{R}^{\dagger} \boldsymbol{P} \boldsymbol{h}_{1}\right)$, where $\left.\operatorname{rank}\left(\boldsymbol{H}_{R}^{\dagger} \boldsymbol{P} \boldsymbol{h}_{1}\right)=1\right)$. Hence, $B \boldsymbol{h}_{2} \sim \mathcal{C N}\left(0, \boldsymbol{I}_{N_{T}-1}\right)$. More specifically, the probability density function (PDF) of $\left\|\boldsymbol{P} \boldsymbol{h}_{1}\right\|^{2}$ and $\left\|\boldsymbol{B} \boldsymbol{h}_{2}\right\|^{2}$ are given as $f_{\left\|\boldsymbol{P} \boldsymbol{h}_{1}\right\|^{2}}(x)=\frac{x^{N_{R}-M-1}}{\left(N_{R}-M-1\right) !} e^{-x}$ and $f_{\left\|\boldsymbol{B} \boldsymbol{h}_{2}\right\|^{2}}(y)=$ $\frac{y^{N_{T}-2}}{\left(N_{T}-2\right) !} e^{-y}$, respectively.

\section{Outage Probability Analysis}

In this section, the information outage probability of MIMO FD relaying systems with null space projection (ZF/ZF scheme) is investigated. An exact as well as a simpler lowerbound expressions for the the outage probability are derived in closed-form, where the asymptotic high SNR regime is also included, through which the achievable diversity order is obtained. The outage probability is defined as the probability that the instantaneous mutual information, $\mathcal{I}=\log _{2}\left(1+\gamma^{\mathrm{ZF} / \mathrm{ZF}}\right)$, falls below a target rate of $R_{0}$ bits per channel use ${ }^{7}$

$$
\begin{aligned}
\mathrm{P}_{\text {out }}^{\mathrm{ZF}}\left(R_{0}\right) & =\operatorname{Pr}\left(\log _{2}\left(1+\gamma^{\mathrm{ZF} / \mathrm{ZF}}\right)<R_{0}\right) \\
& =F_{\gamma}^{\mathrm{ZF}}\left(\gamma_{T}\right) .
\end{aligned}
$$

where $\gamma_{T}=2^{R_{0}}-1$, and $F_{\gamma}^{\mathrm{ZF}}(\cdot)$ denotes the cumulative distribution function (CDF) of the overall SINR.

The CDF and PDF of the random variables (RVs) $\gamma_{1}^{\mathrm{ZF}}$ and $\gamma_{2}^{\mathrm{ZF}}$ are (respectively) given as

$$
F_{\gamma_{1}}^{\mathrm{ZF}}(x)=1-e^{-\frac{x}{\bar{\gamma}_{1}}} \sum_{k=0}^{N_{R}-M-1} \frac{1}{k !}\left(\frac{x}{\overline{\gamma_{1}}}\right)^{k}, x \geq 0
$$

and

$$
f_{\gamma_{2}}^{\mathrm{ZF}}(y)=\frac{{\overline{\gamma_{2}}}^{-N_{T}+1}}{\left(N_{T}-2\right) !} y^{N_{T}-2} e^{-\frac{y}{\gamma_{2}}}, y \geq 0
$$

\footnotetext{
${ }^{7}$ Note that in contrast to (13), the SINR outage probability can be defined as the probability that the instantaneous overall $\gamma^{\mathrm{ZF} / \mathrm{ZF}}$ falls below a threshold $\gamma_{T} ; \operatorname{Pr}\left(\gamma^{\mathrm{ZF} / \mathrm{ZF}}<\gamma_{T}\right)=F_{\gamma}^{\mathrm{ZF}}\left(\gamma_{T}\right)$. Note that according to (13), $\gamma_{T}$ in the case of HD relaying is given as $\gamma_{T}=2^{2 R_{0}}-1$.
} 


\section{A. Exact Outage Probability}

The CDF of the overall SINR $\gamma^{\mathrm{ZF} / \mathrm{ZF}}$ can be derived as [28, Appendix I]

$$
\begin{aligned}
& F_{\gamma}^{\mathrm{ZF}}\left(\gamma_{T}\right)=\operatorname{Pr}\left(\frac{\gamma_{1}^{\mathrm{ZF}} \gamma_{2}^{\mathrm{ZF}}}{\gamma_{1}^{\mathrm{ZF}}+\gamma_{2}^{\mathrm{ZF}}+1}<\gamma_{T}\right) \\
& =1-\int_{0}^{\infty} \overline{F_{\gamma_{1}}^{\mathrm{ZF}}}\left(\frac{\gamma_{T}\left(\gamma_{T}+w+1\right)}{w}\right) f_{\gamma_{2}}^{\mathrm{ZF}}\left(\gamma_{T}+w\right) d w
\end{aligned}
$$

where $\overline{F_{\gamma_{1}}^{\mathrm{ZF}}}(\cdot)$ is the complementary CDF of $\gamma_{1}^{\mathrm{ZF}}$.

Proposition 2. The exact outage probability of the overall SINR $\gamma^{\mathrm{ZF} / \mathrm{ZF}}$ for MIMO FD relaying systems with $M$ cochannel interferers and receive and transmit $Z F$ scheme, can be derived as show in (17) at the top of next page, where $\mathcal{K}_{v}(z)$ is the modified bessel function of the second kind of order $v$.

Proof: The proof is given in Appendix B.

\section{B. Lower-Bound Outage Probability}

Note that though proposition 2 provides an efficient means for analyzing the exact outage probability of the system, this formula is quite complicated as it does not provide simple insights onto the performance analysis of the system. Therefore, in order to gain a better insights onto the performance analysis of the system. The overall SINR in (11) can be tightly upper-bounded by ${ }^{8}$

$$
\gamma^{\mathrm{ZF} / \mathrm{ZF}} \leq \gamma_{\mathrm{up}}^{\mathrm{ZF} / \mathrm{ZF}}=\min \left(\gamma_{1}^{\mathrm{ZF}}, \gamma_{2}^{\mathrm{ZF}}\right)
$$

Therefore, the lower-bound outage probability expression of the overall SINR $\gamma^{\mathrm{ZF} / \mathrm{ZF}}$ (exact for the outage probability of the overall SINR upper-bound $\gamma_{\mathrm{up}}^{\mathrm{ZF} / \mathrm{ZF}}$ ) for the MIMO FD relaying system with $\mathrm{ZF} / \mathrm{ZF}$ scheme can be expressed as

$$
F_{\gamma_{\mathrm{up}}}^{\mathrm{ZF}}\left(\gamma_{T}\right)=1-\frac{\Gamma\left(N_{R}-M, \frac{\gamma_{T}}{\gamma_{1}}\right)}{\Gamma\left(N_{R}-M\right)} \frac{\Gamma\left(N_{T}-1, \frac{\gamma_{T}}{\gamma_{2}}\right)}{\Gamma\left(N_{T}-1\right)} .
$$

Proof: The proof is given in Appendix C.

Next, the lower-bound outage probability expression (19) is then used to derive the asymtotic outage probability of MIMO FD relaying systems with ZF/ZF scheme.

1) Asymptotic Analysis: To validate and characterize the achievable diversity order of the MIMO FD relaying systems with $\mathrm{CCI}$ and $\mathrm{ZF} / \mathrm{ZF}$ scheme at the AF relay, we approximate (19) in the asymptotic high SNR regime assuming $\overline{\gamma_{2}}=\kappa \overline{\gamma_{1}}$, with $\kappa$ denotes a finite constant number and $\overline{\gamma_{1}} \rightarrow \infty$. Hence, the asymptotic outage probability of the overall SINR $\gamma^{\mathrm{ZF} / \mathrm{ZF}}$

\footnotetext{
${ }^{8}$ Note that in order to simplify the mathematical tractability as to gain a better insights onto the performance analysis of the system, it is well known in the conventional half-duplex relaying literature that the overall SNR $\gamma=\frac{\gamma_{1} \gamma_{2}}{\gamma_{1}+\gamma_{2}+1}$ can be tightly upper-bounded by $\frac{\gamma_{1} \gamma_{2}}{\gamma_{1}+\gamma_{2}}$ (see e.g., [29, Eq. (6)]). In addition, the upper-bound $\frac{\gamma_{1} \gamma_{2}}{\gamma_{1}+\gamma_{2}}$ can be further upper-bounded by $\min \left(\gamma_{1}, \gamma_{2}\right)$ (see e.g., [22, Eq. (8)]). It is to be emphasized that the asymptotic results of these bounds are exact.
}

can be expressed as

$$
\begin{gathered}
F_{\gamma}^{\infty}\left(\gamma_{T}\right)=\frac{\left(\frac{\gamma_{T}}{\bar{\gamma}_{1}}\right)^{N_{R}-M}}{\Gamma\left(N_{R}-M+1\right)}+\frac{\left(\frac{\gamma_{T}}{\bar{\gamma}_{1}}\right)^{N_{T}-1}}{\Gamma\left(N_{T}\right)}\left(\frac{1}{\kappa}\right)^{N_{T}-1} \\
+O\left(\left(\frac{\gamma_{T}}{\overline{\gamma_{1}}}\right)^{\min \left(N_{R}-M+1, N_{T}\right)}\right)
\end{gathered}
$$

Proof: The proof is given in Appendix D.

Equation (20) can be further simplified as in (21), shown at the top of next page.

It is straight forward to show from (21) that the achievable diversity order of the MIMO FD relaying systems with ZF/ZF scheme is $\min \left(N_{R}-M, N_{T}-1\right)$.

\section{ERgodic CAPACITy ANALysis}

The capacity analysis is an important performance measure for any wireless communication system as it results in the maximum achievable rate. In this section, we present a rigorous investigation on the ergodic capacity of MIMO FD-AF relaying systems with ZF/ZF scheme. The ergodic capacity is defined as the expected value of the instantaneous mutual information between the source and destination, and is given by

$$
\begin{aligned}
C^{\mathrm{ZF}} & =\frac{1}{\ln 2} \mathbb{E}\left[\ln \left(1+\gamma^{\mathrm{ZF} / \mathrm{ZF}}\right)\right] \\
& =\frac{1}{\ln 2} \mathbb{E}\left[\ln \left(1+\frac{\gamma_{1}^{\mathrm{ZF}} \gamma_{2}^{\mathrm{ZF}}}{\gamma_{1}^{\mathrm{ZF}}+\gamma_{2}^{\mathrm{ZF}}+1}\right)\right] .
\end{aligned}
$$

In order to reduce the required computational complexity, we rely on the following lemma to transform (22) into a more convenient form which facilitates the calculation of the required average by using known results of moment generating functions (MGFs).

Lemma 3. For any $X \geq 0, Y \geq 0$, and $X$ is independent of $Y$, we have [30, Lemma 1]

$$
\begin{aligned}
\mathbb{E}_{X, Y} & {\left[\ln \left(1+\frac{X Y}{X+Y+1}\right)\right] } \\
& =\int_{0}^{\infty} \frac{1}{z}\left(1-\mathcal{M}_{X}(z)\right)\left(1-\mathcal{M}_{Y}(z)\right) e^{-z} d z .
\end{aligned}
$$

where $\mathcal{M}_{X}(z)=\mathbb{E}_{X}\left(e^{-z X}\right)$ denotes the MGF of the $R V X$, and $\mathcal{M}_{Y}(z)=\mathbb{E}_{Y}\left(e^{-z Y}\right)$ denotes the $M G F$ of the $R V Y$.

Once letting $X=\gamma_{1}^{\mathrm{ZF}}$ and $Y=\gamma_{2}^{\mathrm{ZF}}$, the MGFs of $\gamma_{i}^{\mathrm{ZF}}$ is given as [30, Eq. (16)]

$$
\mathcal{M}_{\gamma_{i}}^{\mathrm{ZF}}(z)=\left(\frac{1}{1+\overline{\gamma_{i}} z}\right)^{N_{i}}
$$

where $i \in\{1,2\}, N_{1}=N_{R}-M$ and $N_{2}=N_{T}-1$.

Proposition 4. The exact closed-form ergodic capacity expression of the overall SINR $\gamma^{\mathrm{ZF} / \mathrm{ZF}}$ for the MIMO full-duplex relaying systems with $M C C I$ interferers and ZF/ZF scheme 


$$
\begin{array}{r}
F_{\gamma}^{\mathrm{ZF}}\left(\gamma_{T}\right)=1-\frac{2 e^{-\gamma_{T}\left(\frac{1}{\gamma_{1}}+\frac{1}{\gamma_{2}}\right)}}{\left(N_{T}-2\right) ! \overline{\gamma_{2}} N_{T}-1} \sum_{k=0}^{N_{R}-M-1} \frac{1}{k !}\left(\frac{\gamma_{T}}{\overline{\gamma_{1}}}\right)^{k} \sum_{n=0}^{k}\left(\begin{array}{c}
k \\
n
\end{array}\right)\left(\gamma_{T}+1\right)^{n} \sum_{m=0}^{N_{T}-2}\left(\begin{array}{c}
N_{T}-2 \\
m
\end{array}\right) \gamma_{T}^{N_{T}-m-2} \\
\times\left(\frac{\gamma_{T} \overline{\gamma_{2}}\left(\gamma_{T}+1\right)}{\overline{\gamma_{1}}}\right)^{\frac{m-n+1}{2}} \mathcal{K}_{m-n+1}\left(2 \sqrt{\frac{\gamma_{T}\left(\gamma_{T}+1\right)}{\overline{\gamma_{1} \gamma_{2}}}}\right) .
\end{array}
$$

$$
F_{\gamma}^{\infty}\left(\gamma_{T}\right)= \begin{cases}\frac{\left(\frac{\gamma_{T}}{\gamma_{1}}\right)^{N_{R}-M}}{\Gamma\left(N_{R}-M+1\right)} & , N_{R}-M<N_{T}-1 \\ \frac{\left(\frac{\gamma_{T}}{\gamma_{1}}\right)^{N_{R}-M}}{\Gamma\left(N_{R}-M+1\right)}+\frac{\left(\frac{\gamma_{T}}{\gamma_{2}}\right)^{N_{T}-1}}{\Gamma\left(N_{T}\right)} & , N_{R}-M=N_{T}-1 \\ \frac{\left(\frac{\gamma_{T}}{\gamma_{2}}\right)^{N_{T}-1}}{\Gamma\left(N_{T}\right)} & , N_{R}-M>N_{T}-1\end{cases}
$$

at the AF relay can be written as

$$
\begin{aligned}
C^{\mathrm{ZF}}=\frac{\overline{\gamma_{1}}}{\ln 2} \sum_{k=0}^{N_{R}-M-1} \sum_{m=0}^{N_{T}-2} \frac{1}{\Gamma(k+1) \Gamma(m+1)} \\
\quad \times G_{1,[1: 1], 0,[1: 1]}^{1,1,1,1,1}\left(\begin{array}{c|c}
\overline{\gamma_{1}} & -k ;-m \\
\overline{\gamma_{2}} & - \\
& 0 ; 0
\end{array}\right) .
\end{aligned}
$$

where $G_{., .}(. \mid$.$) denotes the Meijer's G-function [31, Eq.$ (9.301)], $G_{1,[1: 1], 0,[1: 1]}^{1,1,1 .}($.$) denotes the extended generalized$ bivariate Meijer's G-function [32].

Proof: The proof is given in Appendix E.

Note that in the special case when $\overline{\gamma_{1}}=\overline{\gamma_{2}}=\bar{\gamma}$, from (47) and with the help of [31, Eq. (9.211.4)], the ergodic capacity expression can be simplified to

$$
\begin{aligned}
C^{\mathrm{ZF}} & =\frac{\bar{\gamma}^{2}}{\ln 2} \sum_{k=0}^{N_{R}-M-1} \sum_{m=0}^{N_{T}-2} \int_{0}^{\infty} z\left(\frac{1}{1+\bar{\gamma} z}\right)^{k+m+2} e^{-z} d z \\
& =\frac{1}{\ln 2} \sum_{k=0}^{N_{R}-M-1} \sum_{m=0}^{N_{T}-2} \Psi\left(2,1-k-m ; \frac{1}{\bar{\gamma}}\right)
\end{aligned}
$$

where $\Psi(\alpha, \gamma ; z)$ is the Tricomi confluent hypergeometric function of the second kind.

\section{A. Lower-Bound Ergodic Capacity}

In order to find a simpler closed-form lower-bound of the ergodic capacity (22), let us first re-write (22) in a simpler alternative form as follows

$$
\begin{aligned}
C^{\mathrm{ZF}} & =\mathbb{E}\left[\log _{2}\left(\frac{\left(1+\gamma_{1}^{\mathrm{ZF}}\right)\left(1+\gamma_{2}^{\mathrm{ZF}}\right)}{\gamma_{1}^{\mathrm{ZF}}+\gamma_{2}^{\mathrm{ZF}}+1}\right)\right] \\
& =C_{\gamma_{1}}^{\mathrm{ZF}}+C_{\gamma_{2}}^{\mathrm{ZF}}-C_{\gamma_{T}}^{\mathrm{ZF}}
\end{aligned}
$$

where $C_{\gamma_{i}}^{\mathrm{ZF}}=\mathbb{E}\left[\log _{2}\left(1+\gamma_{i}^{\mathrm{ZF}}\right)\right]$, for $i \in\{1,2\}$, and $C_{\gamma_{T}}^{\mathrm{ZF}}=\mathbb{E}\left[\log _{2}\left(1+\gamma_{1}^{\mathrm{ZF}}+\gamma_{2}^{\mathrm{ZF}}\right)\right]$. Due to the computational complexity of $C_{\gamma_{T}}^{\mathrm{ZF}}$ which led to the extended generalized bivariate Meijer's G-function in (25), a simpler lower-bound expression can be derived here by applying Jensen's inequality to $C_{\gamma_{T}}^{\mathrm{ZF}}$ as follows

$$
C_{\gamma_{T}}^{\mathrm{ZF}} \leq \log _{2}\left(1+\mathbb{E}\left(\gamma_{1}^{\mathrm{ZF}}\right)+\mathbb{E}\left(\gamma_{2}^{\mathrm{ZF}}\right)\right) .
$$

Therefore, a simpler closed-form lower-bound expression for the ergodic capacity (22) can be expressed as in (29), shown at the top of next page, where $\mathrm{E}_{n}(\cdot)$ is the exponential integral function [33, Eq. (5.1.4)].

Proof: The proof is given in Appendix F.

\section{MMSE/ZF SCHEME}

It is well known that the $\mathrm{ZF}$ scheme compelely eliminates the CCI interferers and hence produces noise amplification at low interferer-to-noise ratio (INR). Meanwhile, MMSE yields an optimum trade-off between CCI interference suppression and noise amplification. Note here that the MMSE scheme requires the knowledge of $\sigma_{R}^{2}$ in addition to the full CSI that $\mathrm{ZF}$ requires (i.e., in addition to $\boldsymbol{h}_{1}, \boldsymbol{h}_{2}, \boldsymbol{H}_{R}$, and $\boldsymbol{g}_{i}$ with $i=1, \ldots, M)$. For simplicity, it is assumed that the interferer signals have the same average power $\mathcal{E}_{i}=\mathcal{E}_{I} \forall i=1, \ldots, M$. Hence, according to [21], the MMSE combiner is given as $\boldsymbol{w}_{R}=\boldsymbol{h}_{1}^{\dagger}\left(\boldsymbol{h}_{1} \boldsymbol{h}_{1}^{\dagger}+\boldsymbol{G} \boldsymbol{G}^{\dagger}+\frac{1}{\rho_{I}} \boldsymbol{I}_{N_{R}}\right)^{-1}$, where $\rho_{I}=\frac{\mathcal{E}_{I}}{\sigma_{R}^{2}}$.

Therefore, the overall SINR of MIMO FD relaying with CCI utilizing MMSE/ZF scheme can be expressed as

$$
\gamma^{\mathrm{MMSE} / \mathrm{ZF}}=\frac{\gamma_{1}^{\mathrm{MMSE}} \gamma_{2}^{\mathrm{ZF}}}{\gamma_{1}^{\mathrm{MMSE}}+\gamma_{2}^{\mathrm{ZF}}+1}
$$

where $\gamma_{1}^{\mathrm{MMSE}}=\frac{\overline{\gamma_{1}}}{\rho_{I}} \boldsymbol{h}_{1}^{\dagger} \boldsymbol{R}^{-1} \boldsymbol{h}_{1}, \boldsymbol{R}=\boldsymbol{G} \boldsymbol{G}^{\dagger}+\frac{1}{\rho_{I}} \boldsymbol{I}_{N_{R}}$, and $\gamma_{2}^{\mathrm{ZF}}=\overline{\gamma_{2}}\left\|\boldsymbol{B} \boldsymbol{h}_{2}\right\|^{2}$.

It is to be emphasized that analyzing the performance of the overall SINR of MMSE/ZF scheme in (30) is much more complicated than that of the overall SINR of $\mathrm{ZF} / \mathrm{ZF}$ scheme in (11). Therefore, only simulation results for the outage probability and ergodic capacity are presented here for comparison purposes.

\section{NumericAl Results}

In this section, we analyze and validate the presented theoretical results with Monte Carlo simulations. In addition, the 


$$
C^{\mathrm{ZF}} \geq \frac{1}{\ln 2} \sum_{k=0}^{N_{R}-M-1} e^{\frac{1}{\gamma_{1}}} \mathrm{E}_{1+k}\left(\frac{1}{\overline{\gamma_{1}}}\right)+\frac{1}{\ln 2} \sum_{k=0}^{N_{T}-2} e^{\frac{1}{\gamma_{2}}} \mathrm{E}_{1+k}\left(\frac{1}{\overline{\gamma_{2}}}\right)-\log _{2}\left(1+\left(N_{R}-M\right) \overline{\gamma_{1}}+\left(N_{T}-1\right) \overline{\gamma_{2}}\right)
$$

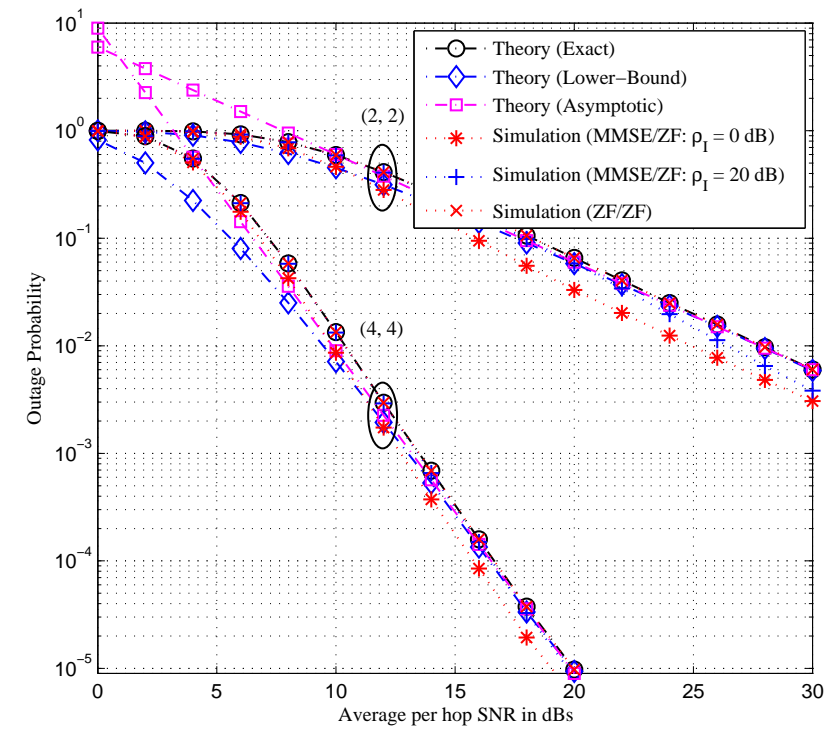

Figure 2: MIMO FD relaying outage probability of $\mathrm{ZF} / \mathrm{ZF}$ and MMSE/ZF schemes against the first-hop SNR $\left(\overline{\gamma_{1}}\right)$ where $\overline{\gamma_{1}}=\overline{\gamma_{2}}$, with $\left(N_{R}, N_{T}\right)$ in the presence of one inteferer $M=1$, where both weak and strong interferers are considered (i.e., $\rho_{I}=0 \mathrm{~dB}$ and $\rho_{I}=20 \mathrm{~dB}$ ).

impact of key system parameters on the outage probability and ergodic capacity are investigated. Without loss of generality, a symmetric settings of per-hop SNR is assumed, i.e., $\overline{\gamma_{1}}=\overline{\gamma_{2}}$. In addition, the source transmission rate is set to $R_{0}=2$ bits/sec/Hz. Hence, the pre-defined SNR threshold is given by ${ }^{9} \gamma_{T}=2^{R_{0}}-1=3$. Besides, in the case of fixed $M, N_{R}$ and $N_{T}$ are denoted in the figures as $\left(N_{R}, N_{T}\right)$. However, in the presence of different $M, N_{R}, N_{T}$ and $M$ are denoted in the figures as $\left(N_{R}, N_{T}, M\right)$. For comparison purposes, results for the case of MIMO HD relaying with CCI and ZF/MRT scheme, i.e., [34, Eq. (16)] for the outage probability analysis and [21, Eq. (20)] for the ergodic capacity analysis, are included with the constraint that the total number of antennas at the relay is $N=N_{R}+N_{T}$. However, as the number of interferers doubles in FD relaying as compared to its HD relaying counterpart, the number of antennas and interferers in the HD relaying is denoted as $\left(N_{R}+N_{T}, \frac{M}{2}\right)$.

In Fig. 2, the outage probability of MIMO FD relaying with $\mathrm{ZF} / \mathrm{ZF}$ and MMSE/ZF schemes against the first-hop SNR is presented for fixed $M$ CCI interferers with weak $\left(\rho_{I}=0\right.$ $\mathrm{dB})$ and strong interferers $\left(\rho_{I}=20 \mathrm{~dB}\right)$, where Monte Carlo simulations of (13) is used to validate the new closed-form exact and lower-bound analytical expressions in (17) and (19), respectively. It is seen that the simulation and proposed

${ }^{9}$ In the case of HD relaying, it is given as $\gamma_{T}=2^{2 R_{0}}-1=15$.

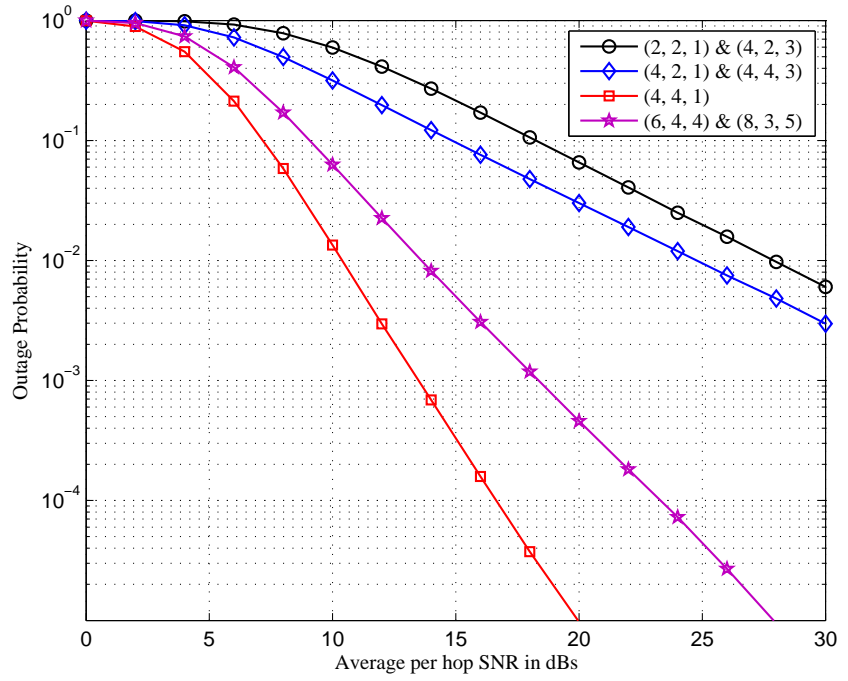

Figure 3: MIMO FD relaying outage probability against the first-hop $\operatorname{SNR}\left(\overline{\gamma_{1}}\right)$ where $\overline{\gamma_{1}}=\overline{\gamma_{2}}$, with $\left(N_{R}, N_{T}, M\right)$.

analytical expression in (17) provide a perfect match which corroborate the exactness of the proposed exact closed-form analytical expression. In addition, the tightness of the proposed lower-bound analytical expression in (19) is also verified, where it becomes exact at high SNR. Our analysis reveals an interesting observation which is beneficial for designing a system. The achievable diversity orders in Fig. 2, which is given by $\min \left(N_{R}-M, N_{T}-1\right)$, are one and three. As a result, it is clearly seen that for fixed $M$, increasing $N_{R}$ and $N_{T}$ yields a significant outage probability improvement due to the increase in diversity order. It is also seen that the outage probability of MMSE/ZF scheme become closer to that of the $\mathrm{ZF} / \mathrm{ZF}$ scheme as the interferer-to-noise ratio $\rho_{I}$ gets higher.

Fig. 3 shows the outage probability for different antenna configurations and number of interferers. It is seen that the diversity order has a higher impact on the outage probability improvement compared to the case of array gain. For instance, the settings $(2,2,1)$ and $(4,2,1)$ have the same diversity order of one. However, the latter has slightly superior performance as a result of higher array gain. Meanwhile, the settings $(2,2,1)$ and $(4,4,1)$ have a diversity order of one and three, respectively. Consequently, the latter enjoys a significant outage performance improvement. Note that in the case of equal per-hop SNR, i.e., $\overline{\gamma_{1}}=\overline{\gamma_{2}}$, system settings with the same diversity order and array gain (degrees of freedom) have the same performance. For example, $(4,2,1)$ and $(4,4,3)$ have the same outage performance. Therefore, for superior system performance, a system designer has to carefully select $N_{R}$ and $N_{T}$ so as to gain the highest possible diversity order, i.e., choosing $N_{R}$ and $N_{T}$ such that $N_{R}-M \approx N_{T}-1$. 


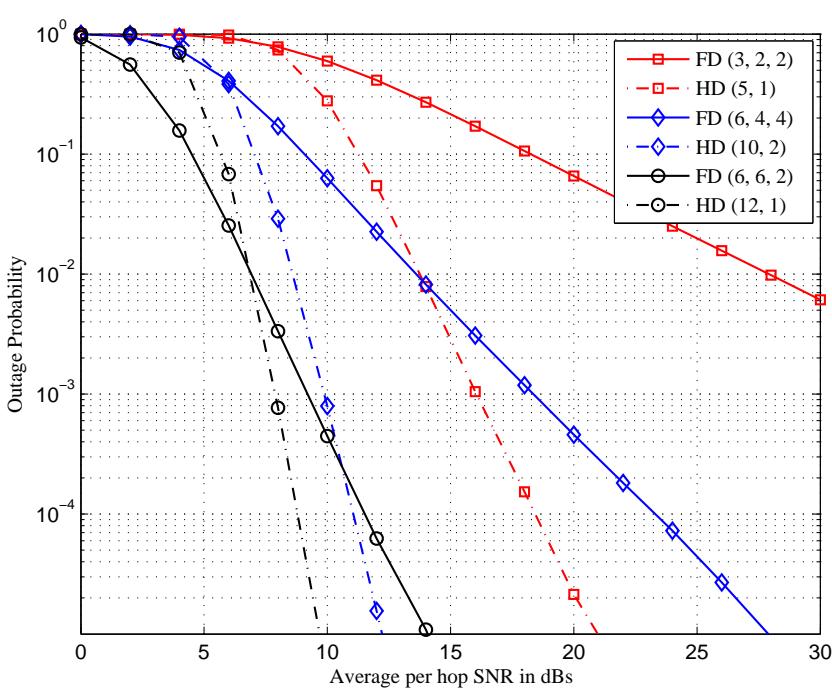

Figure 4: MIMO FD and HD relaying outage probability against the first-hop $\operatorname{SNR}\left(\overline{\gamma_{1}}\right)$ where $\overline{\gamma_{1}}=\overline{\gamma_{2}}$, with $\left(N_{R}, N_{T}, M\right)$ in the FD relaying case, and $\left(N_{R}+N_{T}, \frac{M}{2}\right)$ in the HD relaying case.

In Fig. 4, a comparison between the outage probability of MIMO FD and HD relaying systems is presented. We refer to the so called RF chain preserved condition, where the number of HD relay antennas is $N=N_{R}+N_{T}$. In addition, it is assumed here as stated in [2] that the number of interferers in FD systems is doubled compared to its HD systems counterpart. It is seen that the outage probability of FD relaying mode outperforms that of the HD relaying mode at low SNR. However, at high SNR, the outage probability of HD relaying mode is superior to that of the FD relaying mode. The detrimental effect of the number of interferers on the outage probability performance of FD relaying is clearly observed. Therefore, a hybrid MIMO FD/HD relaying mode may be recommended for superior system performance, where a simple sub-optimal mode selection algorithm could be that in the case of low diversity order, high SNR or high number of interferers $M$, the system is switched to the HD mode, otherwise, it works in the FD mode. The outage probability outperform region of FD relaying can be derived by solving $\mathrm{P}_{\text {out }}^{\mathrm{FD}}\left(R_{0}\right)-\mathrm{P}_{\text {out }}^{\mathrm{HD}}\left(R_{0}\right) \leq 0$. Note that the outage probability expression for a hybrid relaying mode that switches to the appropriate mode according to the instantaneous CSI is given by ${ }^{10}$

$$
\mathrm{P}_{\text {out }}^{\text {Hybrid }}\left(R_{0}\right)=\operatorname{Pr}\left(\max \left(C_{\text {Inst }}^{\mathrm{FD}}, C_{\text {Inst }}^{\mathrm{HD}}\right)<R_{0}\right)
$$

where $C_{\mathrm{Inst}}^{\mathrm{FD}}=\log _{2}\left(1+\gamma^{\mathrm{FD}}\right), C_{\mathrm{Inst}}^{\mathrm{HD}}=\log _{2}\left(\sqrt{1+\gamma^{\mathrm{HD}}}\right)$, $\gamma^{\mathrm{FD}}=\gamma^{\mathrm{ZF} / \mathrm{ZF}}$ is the overall SINR in the case of FD relaying mode, defined in (11), and $\gamma^{\mathrm{HD}}=\gamma^{\mathrm{ZF}}$ is the overall SINR in the case of HD relaying mode, given in [21, Eq. (8)].

\footnotetext{
${ }^{10}$ Note that as far as a closed-form outage probability expression is concern, the analysis of such hybrid relaying mode is a challenging mathematical problem due to the presence of correlation between $\gamma^{\mathrm{HD}}$ and $\gamma^{\mathrm{FD}}$.
}

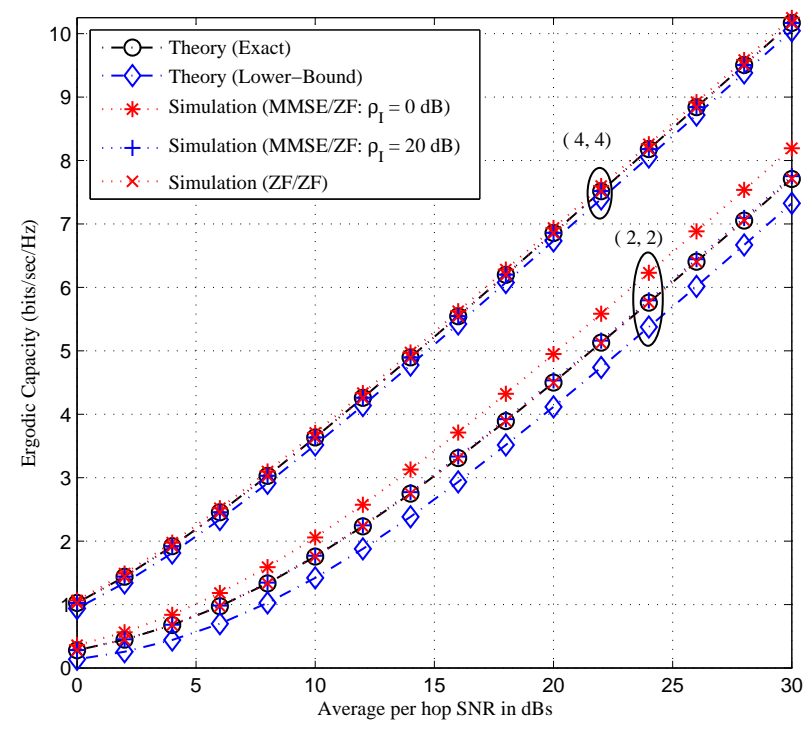

Figure 5: MIMO FD relaying ergodic capacity of ZF/ZF and MMSE/ZF schemes against the first-hop SNR $\left(\overline{\gamma_{1}}\right)$ where $\overline{\gamma_{1}}=$ $\overline{\gamma_{2}}$, with $\left(N_{R}, N_{T}\right)$ in the presence of one inteferer $M=1$, where both weak and strong interferers are considered (i.e., $\rho_{I}=0 \mathrm{~dB}$ and $\left.\rho_{I}=20 \mathrm{~dB}\right)$.

Fig. 5 shows the ergodic capacity of MIMO FD relaying with ZF/ZF and MMSE/ZF schemes against the first-hop SNR for fixed $M$ CCI interferers with weak $\left(\rho_{I}=0 \mathrm{~dB}\right)$ and strong interferers $\left(\rho_{I}=20 \mathrm{~dB}\right)$. Monte Carlo simulations of (22) is used to validate the new closed-form exact and lowerbound analytical expressions in (25) and (29), respectively. It is seen that the simulation and proposed analytical expression in (25) provide a perfect match which corroborate the exactness of the proposed exact closed-form analytical expression. In addition, the tightness of the proposed lower-bound analytical expression in (29) is also verified, where it is shown to improve as $N_{R}$ and $N_{T}$ increase. It is clearly observed that for fixed $M$, increasing $N_{R}$ and $N_{T}$ results in a superior ergodic capacity improvement owing to the increase in diversity order. It is also observed that the ergodic capacity of MMSE/ZF scheme become closer to that of the $\mathrm{ZF} / \mathrm{ZF}$ scheme as $\rho_{I}$ gets higher.

In Fig. 6, the ergodic capacity for different antenna configurations and number of interferers is presented. It is seen that in system settings with equal diversity order, a slight ergodic capacity improvement is observed as a result of higher array gain. However, a significant ergodic capacity improvement is seen as a result of higher diversity order. Note that similarly to Fig. 3 , the settings $(4,2,1)$ and $(4,4,3)$ have the same ergodic capacity as they have the same number of overall equivalent antennas. Likewise, for superior system performance, $N_{R}$ and $N_{T}$ should be chosen as to gain the highest possible diversity order.

Fig. 7 shows a comparison between the ergodic capacity of MIMO FD and HD relaying systems. It is observed that at low diversity order, the ergodic capacity of FD relaying mode is superior to that of the HD relaying mode at high SNR. How- 


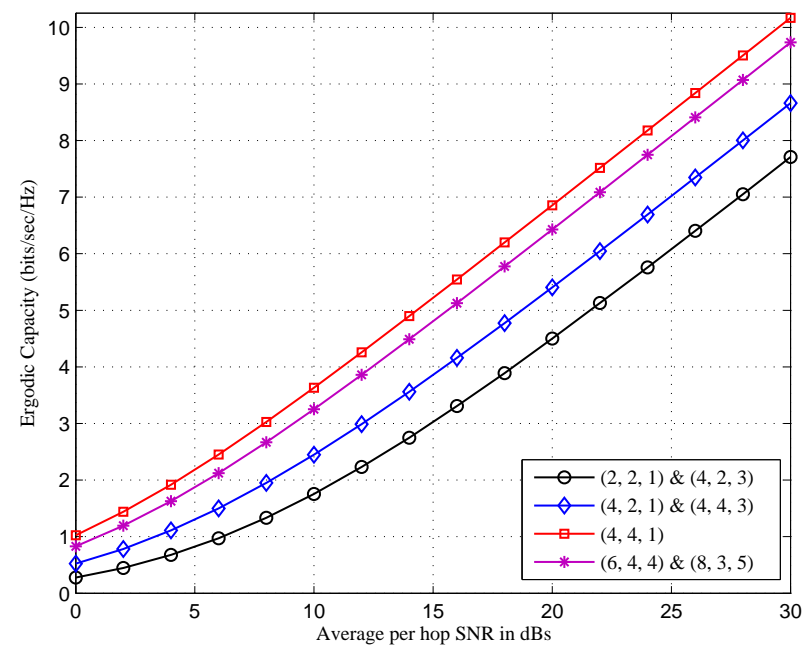

Figure 6: MIMO FD relaying ergodic capacity against the firsthop SNR $\left(\overline{\gamma_{1}}\right)$ where $\overline{\gamma_{1}}=\overline{\gamma_{2}}$, with $\left(N_{R}, N_{T}, M\right)$.

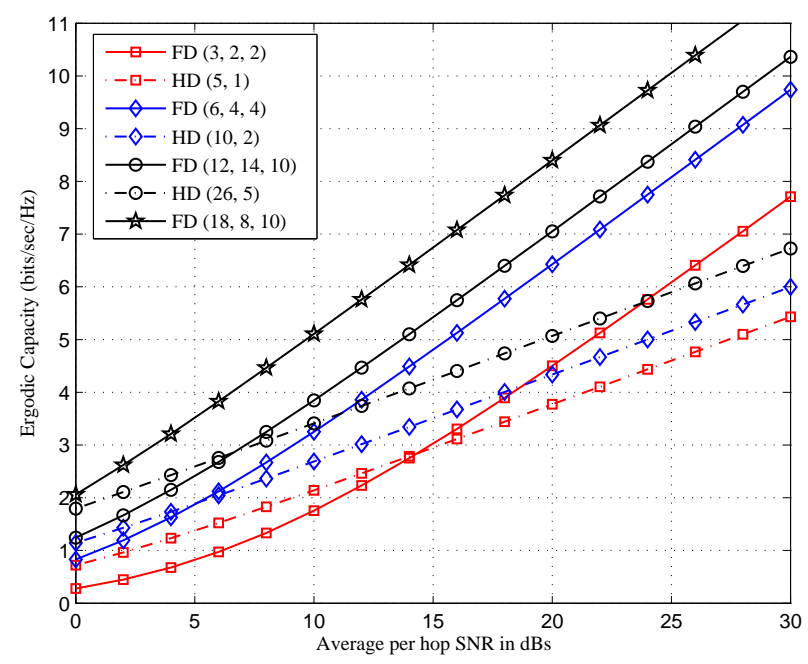

Figure 7: MIMO FD and HD relaying ergodic capacity against the first-hop SNR $\left(\overline{\gamma_{1}}\right)$ where $\overline{\gamma_{1}}=\overline{\gamma_{2}}$, with $\left(N_{R}, N_{T}, M\right)$ in the FD relaying case, and $\left(N_{R}+N_{T}, \frac{M}{2}\right)$ in the HD relaying case.

ever, at low SNR, the ergodic capacity of HD relaying mode outperforms that of the FD relaying mode. The detrimental effect of the number of interferers on the ergodic capacity performance of FD relaying is clearly seen. Meanwhile, at high diversity order, i.e., high $\min \left(N_{R}-M, N_{T}-1\right)$, the performance of FD relaying mode is superior to its HD relaying counterpart at all SNR values. To validate this, let us assume that we have $N_{R}+N_{T}=26$ antennas and $M=10$ $\mathrm{CCI}$ at the FD relay, allocated according to the settings $(12,14,10)$ and $(18,8,10)$. Evidently the latter outperforms the HD relaying mode at all SNR values owing to the high achievable diversity order of 7 , while the former is superior to the HD relaying mode at high SNR due to the low achievable diversity order of 2 . Therefore, for systems with low diversity order, a hybrid MIMO FD/HD relaying mode is recommended for superior system performance, where a simple sub-optimal mode selection scheme is that at low SNR, the system is switched to the HD relaying mode, otherwise, it works in the FD relaying mode. The ergodic capacity outperform region of $\mathrm{FD}$ relaying can be derived by solving $C^{\mathrm{FD}}-C^{\mathrm{HD}} \geq 0$. Note that a hybrid relaying mode which switches to the appropriate mode according to the instantaneous CSI is expressed as ${ }^{11}$ [27]

$$
C_{\text {Inst }}^{\mathrm{Hybrid}}=\max \left(C_{\text {Inst }}^{\mathrm{FD}}, C_{\text {Inst }}^{\mathrm{HD}}\right) .
$$

Therefore, according to the instantaneous CSI which assumed constant at any given coherence time, the FD relaying mode outperforms the HD relaying mode iff $\gamma^{\mathrm{FD}}>$ $\sqrt{\gamma^{\mathrm{HD}}+1}-1$, otherwise, the opposite is true.

\section{CONCLUSIONS}

In this paper, the performance of MIMO full-duplex relaying systems with co-channel interference is investigated, where the ZF/ZF scheme is applied at the amplify-and-forward relay to suppress the co-channel interference and loopback self-interference. Our analysis achieved closed-form results, through which several interesting system insights have been revealed. Exact and tight lower-bound analytical expressions for the outage probability and ergodic capacity were derived in closed-form. In addition, the asymptotic high SNR outage probability is also included to unveil the achievable diversity order of the system. These expressions provide an efficient means for the evaluation of the outage probability and ergodic capacity of MIMO FD relaying systems with co-channel interference. Therefore, without resorting to the time consuming Monte Carlo simulations, the impact of key system parameters such as the number of relay receive and transmit antennas, the number of interferers, and the source transmit power on the system performance are investigated. It is observed that the performances of the MMSE/ZF scheme and ZF/ZF scheme coincide in the asymptotically high interference-to-noise ratio regime. Our analysis reveals that although the number of cochannel interferers is doubled in full-duplex relaying systems compared to the conventional half-duplex relaying systems, full-duplex relaying can significantly improve the system performance.

\section{APPENDIX A}

\section{PROOF OF PROPOSITION 1}

Let us first consider the following constraint optimization problem

$$
\begin{array}{cc}
\boldsymbol{w}^{\star}=\underset{\boldsymbol{w}}{\arg \max } & \left\|\boldsymbol{w}^{\dagger} \boldsymbol{h}\right\|^{2} \\
\text { s.t. } & \boldsymbol{w}^{\dagger} \boldsymbol{H}=0 \&\|\boldsymbol{w}\|=1
\end{array} .
$$

The required solution may be expressed in the following form $\boldsymbol{w}=\frac{\boldsymbol{P} \boldsymbol{x}}{\|\boldsymbol{P x}\|}$, where both constraints are included in the desired form, namely, 1) the projection matrix $\boldsymbol{P}$ which

\footnotetext{
${ }^{11}$ Note that as far as a closed-form ergodic capacity expression is concern, the analysis of such hybrid relaying mode is a challenging mathematical problem due to the presence of correlation between $\gamma^{\mathrm{HD}}$ and $\gamma^{\mathrm{FD}}$.
} 
assures that $\boldsymbol{w} \perp \boldsymbol{H}$ (owing to the fact that $\boldsymbol{w}^{\dagger} \boldsymbol{H}=0$ ), hence, $\left.\boldsymbol{P}=\boldsymbol{I}-\boldsymbol{H}\left(\boldsymbol{H}^{\dagger} \boldsymbol{H}\right)^{-1} \boldsymbol{H}^{\dagger}, 2\right)$ the division by $\|\boldsymbol{P} \boldsymbol{x}\|$ assures that the norm of $\boldsymbol{w}$ equals one, $\|\boldsymbol{w}\|=1$. Note that the maximization problem is now over $\boldsymbol{x}$ instead of $\boldsymbol{w}$. Hence, (33) can be re-written as

$$
\boldsymbol{x}^{\star}=\underset{\boldsymbol{x}}{\arg \max } \frac{\left\|\boldsymbol{x}^{\dagger} \boldsymbol{P} \boldsymbol{h}\right\|^{2}}{\boldsymbol{x}^{\dagger} \boldsymbol{P} \boldsymbol{x}} .
$$

Therefore, once differentiating (34) with respect to $x$ and equating the result to zero, we get

$$
\boldsymbol{x}^{\star \dagger} \boldsymbol{P} \boldsymbol{x}^{\star} \boldsymbol{P h}=\boldsymbol{x}^{\star \dagger} \boldsymbol{P h P} \boldsymbol{x}^{\star} .
$$

It is easily seen that the optimal solution for the vector $\boldsymbol{x}$ is ${ }^{12} \boldsymbol{x}^{\star}=\boldsymbol{h}$.

Hence, the optimal weight vector to (33) can be expressed as

$$
\boldsymbol{w}^{\star}=\frac{\boldsymbol{P h}}{\|\boldsymbol{P h}\|} .
$$

Note that similar results have been derived in [35, Proposition 1].

From (7), based on the optimal solution given in (36), it is straight forward to show that $\boldsymbol{w}_{R}^{\star}=\frac{\boldsymbol{P} \boldsymbol{h}_{1}}{\| \boldsymbol{P \boldsymbol { h } _ { 1 } \|}}$ is the optimal solution to the constraint problem in (7), where $\boldsymbol{P}$ is an idempotent projection matrix which assures that $\boldsymbol{w}_{R}^{\star} \perp G$ (due to $\boldsymbol{w}_{R}^{\dagger} \boldsymbol{G}=0$ ), and $\boldsymbol{P}=\boldsymbol{I}-\boldsymbol{G}\left(\boldsymbol{G}^{\dagger} \boldsymbol{G}\right)^{-1} \boldsymbol{G}^{\dagger}$. Note that $\boldsymbol{P} \boldsymbol{h}_{1} \perp(\boldsymbol{I}-\boldsymbol{P}) \boldsymbol{h}_{1}$, where $\boldsymbol{P}^{2}=\boldsymbol{P}$ and $\boldsymbol{P}^{\dagger}=\boldsymbol{P}$.

Meanwhile, from (8), it is easy to show that $\boldsymbol{w}_{T}^{\star}=\frac{\boldsymbol{B} \boldsymbol{h}_{2}}{\left\|\boldsymbol{B} \boldsymbol{h}_{2}\right\|}$ is the optimal solution to the constraint problem in (8), where $\boldsymbol{B}$ is an idempotent projection matrix which assures that $\boldsymbol{w}_{T}^{\star} \perp \boldsymbol{H}_{R}^{\dagger} \boldsymbol{P} \boldsymbol{h}_{1}$ (due to $\boldsymbol{w}_{R}^{\dagger} \boldsymbol{H}_{R} \boldsymbol{w}_{T}=0$, where $\boldsymbol{w}_{R}^{\star}$ is already derived above), and $\boldsymbol{B}=\boldsymbol{I}-\frac{\boldsymbol{H}_{R}^{\dagger} \boldsymbol{P} \boldsymbol{h}_{1}\left(\boldsymbol{H}_{R}^{\dagger} \boldsymbol{P} \boldsymbol{h}_{1}\right)^{\dagger}}{\left(\boldsymbol{H}_{R}^{\dagger} \boldsymbol{P} \boldsymbol{h}_{1}\right)^{\dagger} \boldsymbol{H}_{R}^{\dagger} \boldsymbol{P h} \boldsymbol{h}_{1}}$.

\section{APPENDIX B \\ PROOF OF PROPOSITION 2}

From (16), the CDF of the overall SINR $\gamma^{\mathrm{ZF} / \mathrm{ZF}}$ is given as

$$
\begin{aligned}
F_{\gamma}^{\mathrm{ZF}}\left(\gamma_{T}\right)=1-\int_{0}^{\infty} \overline{F_{\gamma_{1}}}\left(\frac{\gamma_{T}\left(\gamma_{T}+w+1\right)}{w}\right) \\
\quad \times f_{\gamma_{2}}^{\mathrm{ZF}}\left(\gamma_{T}+w\right) d w .
\end{aligned}
$$

Now with the help of the CDF and PDF of the RVs $\gamma_{1}^{\mathrm{ZF}}$ and $\gamma_{2}^{\mathrm{ZF}}$ defined in (14) and (15), we arrive at

$$
\begin{gathered}
F_{\gamma}^{\mathrm{ZF}}\left(\gamma_{T}\right)=1-\int_{0}^{\infty} e^{-\frac{\gamma_{T}\left(\gamma_{T}+w+1\right)}{\bar{\gamma}_{1} w}} \sum_{k=0}^{N_{R}-M-1} \frac{1}{k !} \\
\times\left(\frac{\gamma_{T}\left(\gamma_{T}+w+1\right)}{\overline{\gamma_{1}} w}\right)^{k} \frac{{\frac{\gamma_{2}}{2}}^{N_{T}+1}}{\left(N_{T}-2\right) !}\left(\gamma_{T}+w\right)^{N_{T}-2} \\
\times e^{-\frac{\gamma_{T}+w}{\bar{\gamma}_{2}}} d w .
\end{gathered}
$$

\footnotetext{
${ }^{12}$ Note that this result can be attained directly once applying Cauchy-Schwarz inequality to (34); $\left\|\boldsymbol{x}^{\dagger} \boldsymbol{P} \boldsymbol{h}\right\|^{2} \leq\|\boldsymbol{P} \boldsymbol{x}\|^{2}\|\boldsymbol{h}\|^{2}$, where equality holds if and only if $\boldsymbol{x}$ is proportional to $\boldsymbol{h}$, i.e., $\boldsymbol{x}^{\star}=\boldsymbol{h}$.
}

After some tedious mathematical manipulations, (38) can be expressed as

$$
\begin{aligned}
& F_{\gamma}^{\mathrm{ZF}}\left(\gamma_{T}\right)=1-\frac{e^{-\gamma_{T}\left(\frac{1}{\gamma_{1}}+\frac{1}{\gamma_{2}}\right)}}{\left(N_{T}-2\right) ! \bar{\gamma}^{N_{T}}-1} \sum_{k=0}^{N_{R}-M-1} \frac{1}{k !}\left(\frac{\gamma_{T}}{\overline{\gamma_{1}}}\right)^{k} \\
& \times \int_{0}^{\infty} e^{-\frac{\gamma_{T}\left(\gamma_{T}+1\right)}{\bar{\gamma}_{1} w}-\frac{w}{\gamma_{2}}} w^{-k}\left(\gamma_{T}+w+1\right)^{k}\left(\gamma_{T}+w\right)^{N_{T}-2} d w .
\end{aligned}
$$

Therefore, once applying the binomial expansions $\left(\gamma_{T}+w+1\right)^{k}=\sum_{n=0}^{k}\left(\begin{array}{c}k \\ n\end{array}\right) w^{k-n}\left(\gamma_{T}+1\right)^{n} \quad$ and $\left(\gamma_{T}+w\right)^{N_{T}-2}=\sum_{m=0}^{N_{T}-2}\left(\begin{array}{c}N_{T}-2 \\ m\end{array}\right) \gamma_{T}^{N_{T}-m-2} w^{m}$, equation (39) reduces to

$$
\begin{gathered}
F_{\gamma}^{\mathrm{ZF}}\left(\gamma_{T}\right)=1-\frac{e^{-\gamma_{T}\left(\frac{1}{\gamma_{1}}+\frac{1}{\gamma_{2}}\right)}}{\left(N_{T}-2\right) ! \bar{\gamma}_{2}} \sum_{T}^{N_{T}-1} \sum_{k=0}^{N_{R}-M-1} \frac{1}{k !}\left(\frac{\gamma_{T}}{\overline{\gamma_{1}}}\right)^{k} \\
\times \sum_{n=0}^{k}\left(\begin{array}{c}
k \\
n
\end{array}\right)\left(\gamma_{T}+1\right)^{n} \sum_{m=0}^{N_{T}-2}\left(\begin{array}{c}
N_{T}-2 \\
m
\end{array}\right) \gamma_{T}^{N_{T}-m-2} \\
\times \underbrace{\int_{0}^{\infty} e^{-\frac{\gamma_{T}\left(\frac{\left.\gamma_{T}+1\right)}{\bar{\gamma}_{1}} w\right.}{N^{2}}-\frac{w}{\gamma_{2}}} w^{m-n} d w}_{I_{1}}
\end{gathered}
$$

To this end, the integral $I_{1}$ is solved by utilizing [31, Eq. (3.471.9)], which upon substituting $I_{1}$ into (40), results in (17), that concludes the proof.

\section{APPENDIX C \\ PROOF OF EQUATION (19)}

From the overall SINR upper-bound (18), we have

$$
\begin{gathered}
F_{\gamma_{\text {up }}}^{\mathrm{ZF}}\left(\gamma_{T}\right)=\operatorname{Pr}\left(\min \left(\gamma_{1}^{\mathrm{ZF}}, \gamma_{2}^{\mathrm{ZF}}\right)<\gamma_{T}\right) \\
=1-\left(1-F_{\gamma_{1}}^{\mathrm{ZF}}\left(\gamma_{T}\right)\right)\left(1-F_{\gamma_{2}}^{\mathrm{ZF}}\left(\gamma_{T}\right)\right) \\
=1-e^{-\frac{\gamma_{T}}{\gamma_{1}}} \sum_{k=0}^{N_{R}-M-1} \frac{1}{k !}\left(\frac{\gamma_{T}}{\overline{\gamma_{1}}}\right)^{k} e^{-\frac{\gamma_{T}}{\bar{\gamma}_{2}}} \sum_{m=0}^{N_{T}-2} \frac{1}{m !}\left(\frac{\gamma_{T}}{\overline{\gamma_{2}}}\right)^{m}
\end{gathered}
$$

upon substituting the $\mathrm{CDF}$ of $\gamma_{1}^{\mathrm{ZF}}$ and $\gamma_{2}^{\mathrm{ZF}}$ and utilizing [31, Eq. (8.352.4)], reduces to (19), that concludes the proof.

\section{APPENDIX D \\ PROOF OF EQUATION (20)}

The asymptotic results can be easily obtained once invoking the asymptotic expansion of the incomplete gamma function [31, Eq. (8.354.1)]. Hence, in the high SNR regime where $\overline{\gamma_{2}}=\kappa \overline{\gamma_{1}}$ and $\overline{\gamma_{1}} \rightarrow \infty$, we have

$$
\begin{aligned}
\gamma\left(N_{R}-M,\right. & \left.\frac{\gamma_{T}}{\overline{\gamma_{1}}}\right) \\
& =\frac{\left(\frac{\gamma_{T}}{\bar{\gamma}_{1}}\right)^{N_{R}-M}}{N_{R}-M}+O\left(\left(\frac{\gamma_{T}}{\overline{\gamma_{1}}}\right)^{N_{R}-M+1}\right)
\end{aligned}
$$


where $\gamma\left(N_{R}-M, \frac{\gamma_{T}}{\gamma_{1}}\right)+\Gamma\left(N_{R}-M, \frac{\gamma_{T}}{\gamma_{1}}\right)=$ $\Gamma\left(N_{R}-M\right)$. Therefore,

$$
\begin{aligned}
& \frac{\Gamma\left(N_{R}-M, \frac{\gamma_{T}}{\bar{\gamma}_{1}}\right)}{\Gamma\left(N_{R}-M\right)} \\
& =1-\frac{\left(\frac{\gamma_{T}}{\bar{\gamma}_{1}}\right)^{N_{R}-M}}{\Gamma\left(N_{R}-M+1\right)}+O\left(\left(\frac{\gamma_{T}}{\overline{\gamma_{1}}}\right)^{N_{R}-M+1}\right) .
\end{aligned}
$$

Similarly, we have

$$
\begin{aligned}
& \frac{\Gamma\left(N_{T}-1, \frac{\gamma_{T}}{\gamma_{2}}\right)}{\Gamma\left(N_{T}-1\right)} \\
& =1-\frac{\left(\frac{\gamma_{T}}{\bar{\gamma}_{1}}\right)^{N_{T}-1}}{\Gamma\left(N_{T}\right)}\left(\frac{1}{\kappa}\right)^{N_{T}-1}+O\left(\left(\frac{\gamma_{T}}{\overline{\gamma_{1}}}\right)^{N_{T}}\right) .
\end{aligned}
$$

Substituting (43) and (44) into (19) yields (20), that concludes the proof.

\section{APPENDIX E \\ PROOF OF PROPOSITION 4}

To get a closed-form solution to (23), it is more convenient to use an alternative form for the MGF expressions given in (24). Hence, with the help of the cumulative distribution function (CDF) of $\gamma_{i}^{\mathrm{ZF}}$ in (14) and (15), an alternative form for the MGF in (24) can be derived as

$$
\begin{aligned}
\mathcal{M}_{\gamma_{i}}^{\mathrm{ZF}}(z) & =z \int_{0}^{\infty} e^{-z \gamma_{i}} F_{\gamma_{i}}^{\mathrm{ZF}}(\gamma) d \gamma \\
& =1-z \overline{\gamma_{i}} \sum_{k=0}^{N_{i}-1}\left(\frac{1}{1+\overline{\gamma_{i}} z}\right)^{k+1}
\end{aligned}
$$

where $i \in\{1,2\}, N_{1}=N_{R}-M$, and $N_{2}=N_{T}-1$.

Therefore, from (22)-(23), the ergodic capacity may be defined as

$$
\begin{aligned}
& C^{\mathrm{ZF}}=\frac{1}{\ln 2} \mathbb{E}\left[\ln \left(1+\frac{\gamma_{1}^{\mathrm{ZF}} \gamma_{2}^{\mathrm{ZF}}}{\gamma_{1}^{\mathrm{ZF}}+\gamma_{2}^{\mathrm{ZF}}+1}\right)\right] \\
& =\frac{1}{\ln 2} \int_{0}^{\infty} \frac{1}{z}\left(1-\mathcal{M}_{\gamma_{1}}^{\mathrm{ZF}}(z)\right)\left(1-\mathcal{M}_{\gamma_{2}}^{\mathrm{ZF}}(z)\right) e^{-z} d z .
\end{aligned}
$$

Hence, once substituting $\mathcal{M}_{\gamma_{1}}^{\mathrm{ZF}}(z)$ and $\mathcal{M}_{\gamma_{2}}^{\mathrm{ZF}}(z)$ from (45) into (46), we arrive at

$$
\begin{aligned}
C^{\mathrm{ZF}} & =\frac{\overline{\gamma_{1}} \overline{\gamma_{2}}}{\ln 2} \sum_{k=0}^{N_{R}-M-1} \sum_{m=0}^{N_{T}-2} \\
& \times \underbrace{\int_{0}^{\infty} z\left(\frac{1}{1+\overline{\gamma_{1}} z}\right)^{k+1}\left(\frac{1}{1+\overline{\gamma_{2}} z}\right)^{m+1} e^{-z} d z}_{I_{2}} .
\end{aligned}
$$

Now, with the aid of the identity

$$
\left(\frac{1}{1+\lambda x}\right)^{\alpha}=\frac{1}{\Gamma(\alpha)} G_{1,1}^{1,1}\left(\lambda x \mid \begin{array}{c}
1-\alpha \\
0
\end{array}\right) .
$$

The integral $I_{2}$ in (47) can be simplified to

$$
\begin{aligned}
I_{2} & =\frac{1}{\Gamma(k+1)} \frac{1}{\Gamma(m+1)} \\
& \times \underbrace{\int_{0}^{\infty} z G_{1,1}^{1,1}\left(\overline{\gamma_{1}} z \mid \begin{array}{c}
-k \\
0
\end{array}\right) G_{1,1}^{1,1}\left(\overline{\gamma_{2}} z \mid \begin{array}{c}
-m \\
0
\end{array}\right) e^{-z} d z}_{I_{3}} \\
= & \frac{1}{\Gamma(k+1)} \frac{1}{\Gamma(m+1)} G_{1,[1: 1], 0,[1: 1]}^{1,1,1,1,1}\left(\begin{array}{c|c}
\overline{\gamma_{1}} & \begin{array}{c}
2 \\
\gamma_{2} \\
\\
0 ; 0
\end{array}
\end{array}\right) .
\end{aligned}
$$

The integral $I_{3}$ is simplified to (49) by utilizing [36, Eq. (2.6.2)]. Now, substituting (49) into (47) yields (25), that concludes the proof.

\section{APPENDIX F \\ PROOF OF EQUATION (29)}

The evaluation of $C_{\gamma_{i}}^{\mathrm{ZF}}=\mathbb{E}\left[\log _{2}\left(1+\gamma_{i}^{\mathrm{ZF}}\right)\right]$ can be done by utilizing [37, Lemma 1] which says, for any $x \geq 0$, we have

$$
\mathbb{E}\left[\ln \left(1+\gamma_{i}^{\mathrm{ZF}}\right)\right]=\int_{0}^{\infty} \frac{1}{z}\left(1-\mathcal{M}_{\gamma_{i}}^{\mathrm{ZF}}(z)\right) e^{-z} d z
$$

Note that in order to attain a closed-form expression to the integral in (50), the alternative form for the MGF of $\gamma_{i}^{\mathrm{ZF}}$ derived in (45) is used. Therefore, upon substituting the MGF (45) into (50), we arrive at

$$
\begin{aligned}
\mathbb{E}\left[\ln \left(1+\gamma_{i}^{\mathrm{ZF}}\right)\right] & =\overline{\gamma_{i}} \sum_{k=0}^{N_{i}-1} \int_{0}^{\infty}\left(\frac{1}{1+\overline{\gamma_{i}} z}\right)^{k+1} e^{-z} d z \\
& =\sum_{k=0}^{N_{i}-1} \Psi\left(1,1-k ; \frac{1}{\overline{\gamma_{i}}}\right) \\
& =\sum_{k=0}^{N_{i}-1} e^{\frac{1}{\bar{\gamma}_{i}}} \mathrm{E}_{1+k}\left(\frac{1}{\overline{\gamma_{i}}}\right) .
\end{aligned}
$$

The integral in (51) is solved by utilizing [31, Eq. (9.211.4)], which upon simplifying $\Psi\left(1,1-k ; \frac{1}{\gamma_{i}}\right)$ to $e^{\frac{1}{\gamma_{i}}} \mathrm{E}_{1+k}\left(\frac{1}{\gamma_{i}}\right)$, reduces to (53), that concludes the proof.

The expectations in (28) can be easily solved by utilizing [31, Eq. (3.381.4)], as follows

$$
\begin{aligned}
\mathbb{E}\left[\gamma_{1}^{\mathrm{ZF}}\right] & =\overline{\gamma_{1}} \int_{0}^{\infty} \frac{x^{N_{R}-M}}{\Gamma\left(N_{R}-M\right)} e^{-x} d x \\
& =\left(N_{R}-M\right) \overline{\gamma_{1}}
\end{aligned}
$$

and

$$
\begin{aligned}
\mathbb{E}\left[\gamma_{2}^{\mathrm{ZF}}\right] & =\overline{\gamma_{2}} \int_{0}^{\infty} \frac{y^{N_{T}-1}}{\Gamma\left(N_{T}-1\right)} e^{-y} d y \\
& =\left(N_{T}-1\right) \overline{\gamma_{2}} .
\end{aligned}
$$




\section{REFERENCES}

[1] M. Duarte, C. Dick, and A. Sabharwal, "Experiment-driven characterization of full-duplex wireless systems," IEEE Trans. Wireless Commun., vol. 11, no. 12, pp. 4296-4307, Dec. 2012.

[2] S. Goyal, P. Liu, S. Panwar, R. Difazio, R. Yang, and E. Bala, "Full duplex cellular systems: will doubling interference prevent doubling capacity?" IEEE Commun. Mag., vol. 53, no. 5, pp. 121-127, May 2015.

[3] D. Kim, H. Lee, and D. Hong, "A survey of in-band full-duplex transmission: From the perspective of PHY and MAC layers," IEEE Commun. Surveys Tuts., Dec. 2015.

[4] Y. Liao, L. Song, Z. Han, and Y. Li, "Full duplex cognitive radio: a new design paradigm for enhancing spectrum usage," IEEE Commun. Mag., vol. 53, no. 5, pp. 138-145, May 2015.

[5] G. Liu, F. Yu, H. Ji, V. Leung, and X. Li, "In-band full-duplex relaying: A survey, research issues and challenges," IEEE Commun. Surveys Tuts., Dec. 2015.

[6] A. Sabharwal, P. Schniter, D. Guo, D. Bliss, S. Rangarajan, and R. Wichman, "In-band full-duplex wireless: Challenges and opportunities," IEEE J. Sel. Areas Commun., vol. 32, no. 9, pp. 1637-1652, Sept. 2014.

[7] H. Cui, M. Ma, L. Song, and B. Jiao, "Relay selection for two-way full duplex relay networks with amplify-and-forward protocol," IEEE Trans. Wireless Commun., vol. 13, no. 7, pp. 3768-3777, July 2014.

[8] I. Krikidis, H. Suraweera, P. Smith, and C. Yuen, "Full-duplex relay selection for amplify-and-forward cooperative networks," IEEE Trans. Wireless Commun., vol. 11, no. 12, pp. 4381-4393, Dec. 2012.

[9] H. Q. Ngo, H. Suraweera, M. Matthaiou, and E. Larsson, "Multipair full-duplex relaying with massive arrays and linear processing," IEEE J. Sel. Areas Commun., vol. 32, no. 9, pp. 1721-1737, Sept. 2014.

[10] T. Riihonen, S. Werner, and R. Wichman, "Mitigation of loopback selfinterference in full-duplex MIMO relays," IEEE Trans. Signal Process., vol. 59, no. 12, pp. 5983-5993, Dec. 2011.

[11] H. Suraweera, I. Krikidis, G. Zheng, C. Yuen, and P. Smith, "Lowcomplexity end-to-end performance optimization in MIMO full-duplex relay systems," IEEE Trans. Wireless Commun., vol. 13, no. 2, pp. 913927, Feb. 2014.

[12] K. Yang, H. Cui, L. Song, and Y. Li, "Efficient full-duplex relaying with joint antenna-relay selection and self-interference suppression," IEEE Trans. Wireless Commun., vol. 14, no. 7, pp. 3991-4005, July 2015.

[13] M. Zhou, L. Song, Y. Li, and X. Li, "Simultaneous bidirectional link selection in full duplex MIMO systems," IEEE Trans. Wireless Commun., vol. 14, no. 7, pp. 4052-4062, July 2015.

[14] D. Nguyen, L.-N. Tran, P. Pirinen, and M. Latva-aho, "On the spectral efficiency of full-duplex small cell wireless systems," IEEE Trans. Wireless Commun., Sept. 2014.

[15] Z. Zhang, X. Chai, K. Long, A. Vasilakos, and L. Hanzo, "Full duplex techniques for 5G networks: self-interference cancellation, protocol design, and relay selection," IEEE Commun. Mag., vol. 53, no. 5, pp. 128-137, May 2015.

[16] $\mathrm{H}$. Ju, E. Oh, and D. Hong, "Improving efficiency of resource usage in two-hop full duplex relay systems based on resource sharing and interference cancellation," IEEE Trans. Wireless Commun., vol. 8, no. 8, pp. 3933-3938, Aug. 2009.

[17] Y. Y. Kang and J. H. Cho, "Capacity of MIMO wireless channel with full-duplex amplify-and-forward relay,' in Proc. IEEE PIMRC '09, Sept. 2009, pp. 117-121.

[18] U. Ugurlu, T. Riihonen, and R. Wichman, "Optimized in-band fullduplex MIMO relay under single-stream transmission," IEEE Trans. Veh. Technol., Jan. 2016.

[19] M. A. Ahmed, C. C. Tsimenidis, and A. F. A. Rawi, "Performance analysis of full-duplex-MRC-MIMO with self-interference cancellation using null-space-projection," IEEE Trans. Signal Process., June 2016.

[20] Z. Cheng, N. Devroye, and T. Liu, "The degrees of freedom of fullduplex bidirectional interference networks with and without a MIMO relay," IEEE Trans. Wireless Commun., April 2016.

[21] G. Zhu, C. Zhong, H. Suraweera, Z. Zhang, C. Yuen, and R. Yin, "Ergodic capacity comparison of different relay precoding schemes in dual-hop af systems with co-channel interference," IEEE Trans. Commun., vol. 62, no. 7, pp. 2314-2328, July 2014.

[22] S. Ikki and S. Aissa, "Performance analysis of two-way amplify-andforward relaying in the presence of co-channel interferences," IEEE Trans. Commun., vol. 60, no. 4, pp. 933-939, April 2012.

[23] I. Trigui, S. Affes, and A. Stephenne, "Ergodic capacity of twohop multiple antenna af systems with co-channel interference," IEEE Wireless Commun. Lett., vol. 4, no. 1, pp. 26-29, Feb. 2015.
[24] H. Alves, C. de Lima, P. Nardelli, R. Demo Souza, and M. Latva-aho, "On the average spectral efficiency of interference-limited full-duplex networks," in Proc. IEEE CROWNCOM '14, June 2014.

[25] H. Alves, R. Souza, D. da Costa, and M. Latva-aho, "Full-duplex relaying systems subject to co-channel interference and noise in nakagami-m fading," in Proc. IEEE VTC '15, May 2015.

[26] G. Sharma, P. Kumar Sharma, and P. Garg, "Performance analysis of full duplex relaying in multicell environment," in Proc. IEEE ICACCI '14, Sept. 2014.

[27] T. Riihonen, S. Werner, and R. Wichman, "Hybrid full-duplex/halfduplex relaying with transmit power adaptation," IEEE Trans. Wireless Commun., vol. 10, no. 9, pp. 3074-3085, Sept. 2011.

[28] G. Amarasuriya, C. Tellambura, and M. Ardakani, "Performance analysis of hop-by-hop beamforming for dual-hop MIMO AF relay networks," IEEE Trans. Commun., vol. 60, no. 7, pp. 1823-1837, July 2012.

[29] M. Hasna and M.-S. Alouini, "End-to-end performance of transmission systems with relays over Rayleigh-fading channels," IEEE Trans. Wireless Commun., vol. 2, no. 6, pp. 1126-1131, Nov. 2003.

[30] A. Almradi and K. Hamdi, "Ergodic capacity analysis of MIMO full duplex relaying with imperfect CSI," in Proc. IEEE GLOBECOM'16, Dec. 2015, pp. 1-6.

[31] I. S. Gradshteyn and I. M. Ryzhik, Tables of Integrals, Series and Products. 6th ed. San Diego, CA, USA: Academic, 2000.

[32] I. Ansari, S. Al-Ahmadi, F. Yilmaz, M.-S. Alouini, and H. Yanikomeroglu, "A new formula for the BER of binary modulations with dual-branch selection over generalized-K," IEEE Trans. Commun., vol. 59, no. 10, pp. 2654-2658, Oct. 2011.

[33] M. Abramowitz and I. A. Stegun, Handbook of Mathematical Functions. New York: Dover, 1965.

[34] G. Zhu, C. Zhong, H. Suraweera, Z. Zhang, and C. Yuen, "Outage probability of dual-hop multiple antenna af systems with linear processing in the presence of co-channel interference," IEEE Trans. Wireless Commun., April 2014.

[35] Z. Ding, K. Leung, D. Goeckel, and D. Towsley, "On the application of cooperative transmission to secrecy communications," IEEE J. Sel. Areas Commun., vol. 30, no. 2, pp. 359-368, Feb. 2012.

[36] A. M. Mathai and R. K. Saxena, The H-Function With Applications in Statistics and Other Disciplines. New York, NY, USA: Wiley, 1978.

[37] K. Hamdi, "Capacity of MRC on correlated rician fading channels," IEEE Trans. Commun., vol. 56, no. 5, pp. 708-711, May 2008.

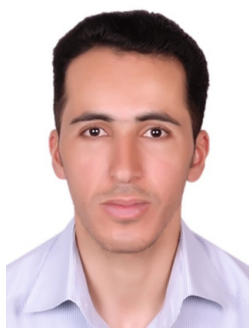

Ahmed Almradi (S'14) received the B.Sc. degree in Electrical and Electronic Engineering in 2004 from the University of Tripoli, Tripoli, Libya, then received the M.Sc. degree in Electrical Engineering in 2012 from Rochester Institute of Technology, Rochester, NY, USA. He is on study leave from Azzaytuna University, Tarhuna, Libya. He is currently working towards the Ph.D degree at the University of Manchester, Manchester, U.K. His research interests are in the modeling, design, and performance analysis of wireless communication systems with special emphasis on MIMO half-duplex and full-duplex relaying systems, wireless information and power transfer (energy harvesting) systems, diversity and beamforming, and OFDM systems.

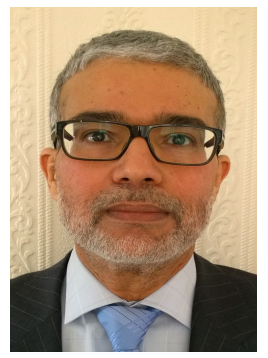

Khairi Ashour Hamdi (M'99-SM'02) graduated from Tripoli, Libya in 1981 with a BSc in Electronics Engineering. He then obtained the MSc (with Distinction) and the $\mathrm{Ph}$. D. in Telecommunication Engineering from Technical University of Budapest in 1988 and 1993, respectively. He was with the University of Essex, Colchester, U.K. He is currently with the School of Electrical and Electronic Engineering, The University of Manchester, Manchester, U.K. His current research interests include modelling and performance analysis of wireless communication systems and networks, green communication systems, and heterogeneous mobile networks. 